Cuadernos de Filología Clásica. Estudios Latinos

ISSN: 1131-9062

http://dx.doi.org/10.5209/CFCL.60935

\title{
Las fuentes hispánicas de los papeles epigráficos de Antonio Agustín (BNE, ms. 5781) ${ }^{1}$
}

\author{
Joan Carbonell Manils²; Gerard González Germain ${ }^{3}$
}

Recibido: 5 de diciembre de 2017 / Aceptado: 5 de junio de 2018

Resumen. A través del análisis de su síloge epigráfica manuscrita (BNE, ms. 5781, ff. 72r-82v; 84r), se identifican las fuentes manuscritas que Antonio Agustín (1517-1585) utilizó para su elaboración, las cuales casi nunca cita e incluso llega a menospreciar: los Epigrammata antiquae urbis Tarraconensis de Lluís Pons d'Icart; la Suma de las inscripciones romanas y memorias de la Bética y la Descripción de la provincia Bethica de Juan Fernández Franco; la síloge de Joan Armengol; el llamado Liber Parmensis o el manuscrito anónimo 5973 de la BNE. El estudio aporta noticias sobre el modus operandi y los intereses epigráficos del arzobispo y se completa con una tabla de correspondencias entre los manuscritos. Palabras clave: Antonio Agustín; epigrafía de tradición manuscrita; humanismo anticuario; Juan Fernández Franco; Lluís Pons d'Icart.

\section{[en] The Spanish sources of Antonio Agustín's epigraphical papers (BNE, ms. 5781)}

\begin{abstract}
Through the analysis of his manuscript collection of inscriptions (BNE, ms. 5781, ff. 72r-82v; 84r), the handwritten sources used by Antonio Agustín (1517-1585) are identified, which the archbishop almost never cites and even undervalues. They are Lluís Pons d'Icart's Epigrammata antiquae urbis Tarraconensis; the Suma de las inscripciones romanas y memorias de la Bética and the Descripción de la provincia Bethica by Juan Fernández Franco; Joan Armengol's sylloge; the so-called Liber Parmensis, and the anonymous ms. 5973 of the BNE. The study also provides information about the modus operandi and the epigraphic interests of the archbishop; it is completed with a table of correspondences between the manuscripts.

Keywords: Antonio Agustín; manuscript tradition of epigraphy; antiquarian humanism; Juan Fernández Franco; Lluís Pons d'Icart.

Sumario: 1. Introducción. 2. Los Epigrammata antiquae urbis Tarraconensis de Lluís Pons d'Icart. 3. Otras fuentes tarraconenses: las síloges de Joan Armengol y de Bux(-?). 4. La epigrafía bética: los manuscritos de Fernández Franco. 5. Otras fuentes utilizadas por Agustín. 6. Conclusiones. 7. Apéndice: tabla comparativa de los Adversaria de Antonio Agustín y sus fuentes. 8. Bibliografía.

Cómo citar: Carbonell Manils, J.; González Germain, G., «Las fuentes hispánicas de los papeles epigráficos de Antonio Agustín (BNE, ms. 5781)», Cuad. Filol. Clás. Estud. Lat. 38.1 (2018), 109-137.

\footnotetext{
1 Este trabajo se ha realizado en el marco del proyecto de investigación financiado por el Ministerio de Economía, Industria y Competitividad dentro del programa de I+D+i FFI2016-77723-P "Influencia de los Epigrammata Antiquae Vrbis (Romae 1521) en el nacimiento de los estudios anticuarios. Estudio y edición de los marginalia de los humanistas del s. XVI".

2 Universitat Autònoma de Barcelona. Email: joan.carbonell@uab.cat

3 Universitat Autònoma de Barcelona. Email: gerardggermain@gmail.com
} 


\section{Introducción}

Como es de sobras conocido por los epigrafistas, el ms. 5781 de la Biblioteca Nacional de España (Carbonell - Gimeno - Vargas 1992; Hernando Sobrino 2009, 222-228) contiene, entre los folios 1-89, una serie de papeles epigráficos (conocidos como Aduersaria) que pertenecieron al ilustre humanista Antonio Agustín (15171586). ${ }^{4}$ Un primer conjunto (ff. 1-69) son copias de inscripciones no hispánicas junto a algunas cartas y papeles varios. Los folios $71 \mathrm{r}-84 \mathrm{v}$ contienen una serie de epígrafes hispánicos copiados casi exclusivamente por el arzobispo, salvo dos inscripciones oscenses debidas a la mano de Felipe Puyvecino de Castro (Carbonell 1993, 114-115 y figs. 1-3); una serie de epígrafes saguntinos atribuibles con toda seguridad a Martín López de Bailo, su secretario tarraconense, y tres epígrafes de Guissona debidos a la mano de Pere Joan Núñez (Carbonell - Gimeno - Vargas 1992, 181).

Los trabajos anteriores se han ocupado de la descripción de los papeles y de la identificación de las inscripciones presentes, pero han dejado de lado un aspecto que nos parece fundamental: el origen de la serie de inscripciones hispánicas contenida en los folios 72r-82v y 84r. ¿Se trata de autopsias del mismo Agustín o -como su formato parece indicar- de la copia de recopilaciones ajenas? En este caso, ¿es el vaciado de una única fuente o de más de una? ¿Podemos identificar esta(s) fuente(s) con alguno de los numerosos manuscritos epigráficos que conocemos actualmente? Nuestra investigación ha dado respuesta a muchas de estas cuestiones, lo que a su vez nos permite arrojar nueva luz sobre la actividad epigráfica del arzobispo y obliga a matizar el valor que se viene atribuyendo a estos papeles desde Hübner hasta hoy.

Desde el punto de vista codicológico, los folios $72 \mathrm{r}-82 \mathrm{v}$ consisten en 11 hojas escritas en recto y verso, cada una doblada longitudinalmente y por separado en forma de cuadernillo, de manera que las inscripciones se distribuyen en cuatro columnas, dos en el recto y dos en el verso [Fig. 1]. ${ }^{5}$ Los folios conservan la numeración original de Agustín (en el margen superior de la segunda columna del recto), que los dividió en dos grupos numerados del 1 al 6 (ff. 72r-77v) y del 1 al 5 (78r-82v) respectivamente; este último lleva el encabezamiento Tarracone y está formado únicamente por epígrafes de esta ciudad. Esta es, pues, la distribución.

\begin{tabular}{|c|c|c|c|}
\hline Numeración Agustín & Foliación actual & Numeración Agustín & Foliación actual \\
\hline 1 & $72 \mathrm{r}-\mathrm{v}$ & 1 & $78 \mathrm{r}-\mathrm{v}$ [Tarracone] \\
\hline 2 & $73 \mathrm{r}-\mathrm{v}$ & 2 & $79 \mathrm{r}-\mathrm{v}$ \\
\hline 3 & $74 \mathrm{r}-\mathrm{v}$ & 3 & $80 \mathrm{r}-\mathrm{v}$ \\
\hline 4 & $75 \mathrm{r}-\mathrm{v}$ & 4 & $81 \mathrm{r}-\mathrm{v}$ \\
\hline 5 & $76 \mathrm{r}-\mathrm{v}$ & 5 & $82 \mathrm{r}-\mathrm{v}$ \\
\hline 6 & $77 \mathrm{r}-\mathrm{v}$ & & \\
\hline
\end{tabular}

El f. 84, en cambio, aparece aislado del resto, escrito solo en el recto y con cuatro dobleces en forma de cruz.

4 De la ingente bibliografía existente relativa a Antonio Agustín, señalamos solo algunos de los trabajos principales dedicados a su labor anticuaria, exceptuando la bibliografía citada en el cuerpo del texto: Crawford 1993; Carbonell 1991; 2002; 2005; 2009; Carbonell - Soler 2011.

5 Así, el orden de lectura original de cada cuadernillo era el siguiente: segunda columna del recto, primera columna del verso, segunda columna del verso y primera columna del recto. 
Observando estos papeles se concluye con facilidad que se trata de un trabajo de copia seguido, realizado en sesiones más o menos continuas a partir de originales en su poder, que Agustín no menciona. Solo la consulta de su epistolario, por una parte, y la colación de los epígrafes con misceláneas manuscritas o publicadas antes del inicio del último cuarto del siglo, nos dan algún indicio sobre las fuentes concretas y la cronología de la copia.

\section{Los Epigrammata antiquae urbis Tarraconensis de Lluís Pons d'Icart}

En un primer trabajo de colación hemos determinado claramente la fuente de los ff. $78 \mathrm{r}-82 \mathrm{v}$, que son los más homogéneos en cuanto a ubicación de los epígrafes ya que todos ellos pertenecen a Tarragona, según reza su encabezamiento. Como recientemente sugerimos (Carbonell - Gimeno 2016, 273), tenemos evidencias definitivas para confirmar que son una copia de los Epigrammata antiquae urbis Tarraconensis de Lluís Pons d'Icart (1518/20-1578), la obra elaborada por el humanista que nunca vio la luz, ${ }^{6}$ y que confeccionó en paralelo a su Libro de las grandezas y cosas memorables de la metropolitana, insigne y famosa ciudad de Tarragona (Lérida 1572).

Pons d'Icart era sobrino de Isabel Agustín, hermana del obispo, casada en primeras nupcias con Cristòfor d'Icart; podemos suponer que su relación empezara ya en 1564, cuando Agustín se desplazó desde Lérida a Tarragona para asistir al concilio provincial. En 1573, alentado por el entonces obispo ilerdense, Icart había publicado en la imprenta de Pedro Robles y Juan de Villanueva un Libro de las grandezas de Tarragona, de gestación lenta, de cuya impresión sabemos que estaba concluida el 31 de enero de 1572 (uid. Duran 1984, 27-29). En esta obra solo publicó 27 epígrafes de Tarragona porque en paralelo, tal como consta en una epístola de 7 de mayo de 1573, se esmeraba por «posar en bell lo libre he interpretations de les pedres scrites per poderlo aportar a la correctio de V(ostra) S(enyoria) que per lo molt li so servidor» (Cahner 1978, II, 85), sin duda los Epigrammata antiquae urbis Tarraconensis.

La tarea de reunir, redactar «en bell» e interpretar los epígrafes se alargó tres años, siendo interferida por la aparición de Las Antiguedades de las ciudades de España (Alcalá de Henares 1575) de Ambrosio de Morales. Esta es la razón por la cual el libro de Icart nunca vio la luz, como se entrevé de sus propias palabras dirigidas a Agustín el 12 de marzo de 1576, en que le comunica «[haver] acabat lo meu [libre] ab ses escolias per ordinem alphabeti y dirigit a v(ostra) s(enyoria) y posat debayx de sa protectio» y le promete que nadie «veura lo meu sino v(ostra) s(enyoria) que ma de fer merce de passarne la llima, perque sens ella li falta la autoritat que a menester» (Cahner 1978, II, 87).

Agustín tuvo en sus manos el nuevo libro casi simultáneamente a la carta mencionada, ya que justo un mes después, el 24 de abril, se apresuraba a escribir a Fulvio Orsini unas duras palabras contra el borrador de Icart y contra otro de epigrafia bé-

\footnotetext{
Se trata del ms. de Wolfenbüttel, Herzog-August-Bibliothek, cod. Guelf. 20.11 Aug. $4^{\circ}$, ff. 52-163v. Existe una segunda parte de la síloge conservada en Barcelona, Biblioteca de Catalunya, ms. 1774, con explicaciones provenientes de las transcripciones del volumen de Wolfenbüttel. Ha tratado sobre la síloge muy recientmente J. Alcina en su ponencia «Martín López de Bailo, secretario de Agustín y corrector de Lluís Pons d'Icart en el ms. de la biblioteca ducal de Wolfenbüttel 20.11 Aug. 4to», presentada en el Simposio Internacional "L'arquebisbe Antonio Agustín en el 500è aniversari del seu naixement" (junio 2017), que verá la luz en el Butlletí de la Reial Academia de Bones Lletres, 2018. También puede consultarse Duran 1984, 41-48; 2005, 161-166; Mayer 1984, 42-43; Massó 1985, 92-98; 1989, 84-86; 2003, 37-41.
} 
tica. Volveremos a este último más adelante. Afirma Agustín: «Un libro d’inscritioni della Bethica ho nelle mani assai ridiculo, et un altro di Tarracona è gia fuori, ma mi vergogno che si vedano in Italia, et così non posso mandarli») (Agustín 1765-1774, VII, 258).

No es la primera vez ni será la última que el humanista menosprecia la calidad de los trabajos de sus compatriotas al compararlos con los de sus ilustrados amigos italianos. Era una práctica habitual en él, que vivió sus obispados hispánicos de Lérida y Tarragona como un verdadero exilio intelectual. ${ }^{7}$

El libro de Icart estaba, pues, en manos del obispo y cabe suponer que fue en ese momento cuando empezó a copiar todos los epígrafes en los folios 78r-82v. A la vista del formato y de la factura del manuscrito de los Epigrammata, se entiende la valoración negativa del obispo, aunque a pesar de esta consideración, el libro fuera literalmente 'fusilado' por él. Así, Agustín sigue exactamente la secuencia del manuscrito de Icart con algunos cambios justificados. Por ejemplo, en el f. 79v (col. II) omite $C I L \mathrm{II}^{2} / 14,1234$ (y la tacha en el manuscrito de Icart) al creerla coincidente con la que acaba de copiar en la columna anterior, que, en realidad, es una inscripción distinta con el mismo texto $\left(C I L \mathrm{II}^{2} / 14,1232\right){ }^{8}$ en el f. $80 \mathrm{r}$ (col. II) omite una fragmentaria (CIL II $\left.4437=\mathrm{II}^{2} / 14,1692\right)$ e incluye $C I L$ II $4337=\mathrm{II}^{2} / 14,1479$ (que aparece más tarde en Icart) al lado de CIL II $4147=\mathrm{II}^{2} / 14,1031$, probablemente a causa de la coincidencia del antropónimo M. Aurelius en ambos textos; en el f. $81 \mathrm{r}$ (col. II) omite seis epígrafes fragmentarios ilegibles; en el mismo folio deja de copiar otros dos epígrafes cristianos por no considerarlos antiguos. ${ }^{9}$

Agustín solo interrumpe la serie de Icart para introducir a) epígrafes tarraconenses de otras fuentes -que Agustín identifica como ex Io. Hermangolio (f. 81v col. II) y ex Hermangolio et Bux. (f. 81r col. I y 82r col. I), a las cuales nos referiremos después- y b) la inscripción en griego $\Delta \mathrm{HMO} / \mathrm{C} \Theta \mathrm{E} / \mathrm{NHC}$ al final del f. $81 \mathrm{v}$ col. I.

Esta inscripción griega $\left(C I L \mathrm{II}^{2} / 14, \mathrm{G} 2\right)$, que forma parte de las pocas referidas por Icart en su Libro de las grandezas ${ }^{10}$ y de cuya existencia, por tanto, Agustín sabía, había despertado un interés singular en él, de modo que en una de sus visitas a Tarragona, siendo obispo de Lérida, relataba a su amigo Orsini el 13 de noviembre de 1574:

Questa scrivo in Tarragona. Sono andato a salutar Demosthene da parte vostra, che se non fossi la disgrazia del naso, saria una bella cosa; è di buona mano et di buona

Años después volvemos a leer una consideración desfavorable respecto de otra obra de Pons d'Icart, Catàlogo dels arquebisbes de Tarragona que tampoco vio la luz. Escribe Agustín a Zurita el 8 de diciembre de 1577: «Yo tengo de remendar unas Constitutiones Provinciales de Tarragona, y al principio esta una lista con parte de la vida de los arçob(isp)os llena de errores en los tiempos y nombres y sucession dellos; y un letrado que ha impresso un libro en loor de Tarrag(on)a, que se dize micer Luis Ponce, ha hecho otro libro de las vidas de los mismos con alguna mayor diligencia, pero no sin errores, y no merece que se imprima por muchas causas que serian largas de contar» (Agustín 1765-1774, VII, 218).

Las dos copias se encuentran en los folios $80 \mathrm{v}$ y $89 \mathrm{v}$ del ms. de Icart.

9 $\quad C I L$ II $^{2} / 14,2121=$ IHC 189 y CIL II²/14, 2132 = ICERV 219 (Wolfenbüttel, Herzog-August-Bibliothek, cod. Guelf. 20.11 Aug. 4º f. 123r-v). En el primer caso, el secretario de Agustín, López de Bailo, ha anotado al margen del texto non est antiqua; en el segundo, es el mismo Icart quien anota «no la tinc per de las antigas». Agustín copiará más adelante (f. 82r col. II) estos dos ejemplares junto con $C I L \mathrm{II}^{2} / 14,1234$.

10 Escribe Pons d'Icart (1572, f. 176r): «Se ha hallado en Tarragona en el huerto de Francisco de Soldevilla cavallero, que esta al puerto fabricado, dentro de aquel antiquissimo edificio que era el templo de Neptuno al subir de la escalera esta puesta en la pared la estatua o figura de Demosthenes de marmol blanco alabastrino y tiene al ombro drecho $[$ sic $]$ estas letras $\triangle \mathrm{HMOC} \Theta E N H C$ que quieren decir Demosthenis»». 
pietra, ma non vedo perché fossi fatto nudo fin alle mammelle e con due haste alle spalle. Le lettere mi paiono pure antique ancora che stanno in luogo poco capace di lettere. Lo edificio dove è conservato è molto antiquo et pare un tempio, o vero un bagno, et si trovano molte inscritioni di statue et di monumenti nel medesimo luogo, che è fuor della città et appresso il mare. La figura è di mezzo rilievo di marmo bianco (Agustín 1765-1774, VII, 257). ${ }^{11}$

\section{Otras fuentes tarraconenses: las síloges de Joan Armengol y de Bux(-?)}

Los Epigrammata de Pons d'Icart no fueron la única fuente de la que se sirvió Agustín para las inscripciones tarraconenses de sus Adversaria. En el f. 81v, después de copiar más de 150 epígrafes de Icart, interrumpe la serie (que recuperará en el f. 82r) e inserta un pequeño conjunto precedido de la indicación ex Io(anne) Hermang(olio). Lo mismo ocurre al final de este cuadernillo (ahora la primera columna del f. 81r), donde en un inicio figuraban epígrafes procedentes de esta fuente, a los que vinieron a sumarse, en un segundo tiempo (como se colige del tono distinto de las tintas), todavía otros de una síloge diferente; Agustín señaló esta doble procedencia bajo el título Tarracone ex Herm. et Bux. Estas dos indicaciones, en efecto, se erigen como las únicas de toda la síloge con las que Agustín identifica la fuente de sus copias.

La primera de estas dos fuentes es Joan Armengol, que aparece citado en diversas ocasiones relacionado con Agustín. En su epistolario, se le menciona en dos cartas de 1539 y 1541 , de las que se conoce que era jurisconsulto, condiscípulo del futuro prelado en Bolonia y que existía entre ellos un grado de amistad íntima. ${ }^{12}$ Una rúbrica en un manuscrito epigráfico de Jean Matal (secretario y colaborador de Agustín en Roma entre 1545 y 1555) lo menciona como originario de Tarragona y responsable de la copia de una serie de inscripciones de la ciudad, hecha con anterioridad a 1546: Inscriptiones Tarraconensium monumentorum collectae ex ipsis saxis ab Ioanne Hermangolio Tarraconensi ex quo descripsit Ant. Augustinus, ego ex Augustino $M D X L V I .^{13}$

No existe ningún estudio sobre Joan Armengol, sus intereses anticuarios o el origen de su síloge. Los Armengol eran una familia noble de Tarragona, quienes obtuvieron en 1515 el señorío de Rocafort de Queralt (Gual Vilà 1992-1993; Rovira i Gómez 2006). De nuestro Joan Armengol sabemos que en agosto de 1552 continuaba en Roma, pues Matal recibió de él el texto de una inscripción (CIL VI 14215) ubicada en el jardín de la residencia del cardenal Rodolfo Pio da Carpi. ${ }^{14}$ Matal usa la

\footnotetext{
G. Alföldy no recoge este interesante testimonio epistolar de Agustín en su edición para el CIL.

12 Vid. Flores Sellés 1980, 83 n 59: Quod Hermangolium Franciscumque Lopem meos summa sis humanitate complexus, ut ex illius sermone huius litteris cognoui (carta de 27 de octubre de 1539 a Diego Hurtado de Mendoza); y p. 104 n ${ }^{\circ}$ 76: Io. Hermangolium et Petrum Ruitium iure consultos quanti faciam nosti; quanti autem facere debeam ego id solus scio: gratius abs te nihil consequi possum quam si utrumque ita iunes ut homines doctos et gratos tibi in perpetuum deuincias. Quod si feceris tum tu me optime ornasse existimato, ceteris in rebus mei memoriam negligas licet (carta de 15 de mayo de 1541 de recomendación a Diego de Albión).

13 Biblioteca Apostolica Vaticana, ms. Vat. lat. 6039, f. 206r. Al margen, Matal añadió: Emendaui fere omnes ex codice Tauerae, multis tamen locis falsissimo. Inde autem licet coniicere non ipsum Hermangolium sed alterum e saxis sumpsisse.

14 Biblioteca Apostolica Vaticana, ms. Vat. lat. 6037, f. 9r: In hortis car(din)alis Carpen(sis). Jo(hannis) Hermangolii manu 1552 Augusto. Cf. Vagenheim 2006, 243.
} 
versión de una inscripción napolitana de la síloge de Armengol para compararla con las versiones de Antoine Morillon, Simon de Vallambert, Louis Budé y Guillaume Philandrier y realizar una revisión crítica (Cooper 1993, 108).

De nuevo Armengol será citado por Agustín, situándolo en Flandes, en sus notas manuscritas conocidas como Alveolus. A propósito de la diferencia de forma entre los cuernos de las vacas y los de los toros, escribe:

Tra li tori et le vache è differenza nelli corni grande, che le vache hanno li corni volti semplicemente verso di se et fanno un semicirculo. Et per questo eranno dii antichi consecrate a la luna, per esserli nelle corni conformi. Quelli delli tori et bovi si voltano di fora in figura di S. Questi sentì dir a Giovanni Hermangolo haverlo imparato in Fiandra et io l'o osservato nelle medaglie et altre antichità et nelli animale istessi (Flores Sellés 1982, 59).

Como era de esperar, las inscripciones procedentes de Armengol en los Adversaria ya aparecen, con las mismas lecciones, en el manuscrito de Matal. Según el francés, Armengol se atribuía las autopsias de su síloge, pero un análisis del conjunto permite observar que la gran mayoría de los textos ya habían sido recogidos en la síloge del tortosino Francesc Vicent (m. 1523) (González Germain 2013, 56-102), en ocasiones con las mismas lecturas erróneas que encontramos en Armengol. ${ }^{15}$

El contar con un testimonio independiente de la síloge de Armengol nos ha permitido distinguir con seguridad las inscripciones del f. $81 \mathrm{r}$ de los Adversaria procedentes de él de aquellas que derivan de la fuente que Agustín denomina con la abreviación "Bux.". A pesar de desconocer el significado de este nombre, sí hemos podido identificar finalmente la fuente en cuestión: se trata del manuscrito epigráfico hoy conocido como Liber Parmensis, que fue escrito en Barcelona en la segunda década del s. XVI y que deriva, en su mayor parte, de la síloge de Pere Miquel Carbonell (1434-1517). ${ }^{16}$ El gran número de errores banales que cometió el autor del Liber Parmensis y que Agustín repite, ${ }^{17}$ así como la coincidencia en una versión de CIL II'2/14 1445 a transmitida únicamente por estos dos manuscritos, confirman que la fuente que Agustín identifica con la abreviatura Bux. es efectivamente el Liber Parmensis. Es, pues, este un paso importante para determinar la autoría de un conjunto a partir de ahora menos anónimo.

\section{La epigrafía bética: los manuscritos de Juan Fernández Franco}

Hemos mencionado antes la opinión de Agustín sobre «un libro d'inscritioni della Bethica [...] assai ridiculo», que había llegado a sus manos en 1576. Al igual que sucede con el libro de Icart, no puede tratarse de unos simples pliegos de papel con

\footnotetext{
Así, por ejemplo, en CIL II $4339=\mathrm{II}^{2} / 14$, 2330: Baene (en lugar de Baebiae) y Aheseus (en lugar de Theseus).

16 Parma, Biblioteca Palatina, Z II 22763; se trata de unos papeles manuscritos encuadernados al final de un ejemplar de los Epigrammata Antiquae Vrbis (Roma 1521) editados por Giacomo Mazzocchi; uid. González Germain 2016.

17 Así, por ejemplo, en CIL II 4304 = II²/14 1262: Laraustali en lugar de Lar. Augustali (así en Carbonell; Girona, Arxiu Capitular, ms. 69, f. 72r); en esta misma inscripción, las dos lecciones incorrectas premisso o potentissimo (en lugar de pientissimo) que presenta Agustín se corresponden con las dos transcripciones del Liber Parmensis (f. $3 \mathrm{r} \mathrm{y} 3 \mathrm{v}$, respectivamente).
} 
una secuencia más o menos extensa de inscripciones: eso no hubiera merecido el calificativo de 'libro' por parte del humanista; se trataría, al contrario, de una pequeña 'monografía' sobre epigrafía bética que habría llegado a sus manos, quizás con la intención por parte de su autor de ser censurada tal como había sucedido con el volumen de Icart. Es indudable que el «libro ridiculo» no pasó el cedazo.

La mención de un libro con epigrafía bética nos ha llevado a pensar directamente en Juan Fernández Franco (1520/5-1601), el único personaje centrado en elaborar conjuntos de este tipo por aquellos tiempos (Gozalbes Cravioto 2007; Carbonell - Gimeno 2016). Cercanas o anteriores a la fecha de la carta existen cuatro monografías manuscritas de Fernández Franco, nunca publicadas: la Suma (1560), ${ }^{18}$ el Monumento (ca. 1565-1567), ${ }^{19}$ la Descripción de la provincia Bethica $(1570)^{20}$ y el Compendio y breve summario de la descripción de la provincia Bethica (1571). ${ }^{21}$

El cotejo de la síloge agustiniana contenida en los Adversaria con cada una de estas obras nos ha dado un doble resultado. Por un lado, la secuencia de inscripciones béticas que copia Agustín en los ff. 72r-74r aparece en el mismo orden que en la Suma de Franco, a pesar de que el obispo omite algunas de ellas. ${ }^{22}$

Pero la Suma no es la única obra de Fernández Franco que Agustín tuvo entre sus manos y que vació en sus Adversaria. La hoja que cierra el primero de los dos cuadernos (f. $77 \mathrm{r}-\mathrm{v}$ ) contiene de principio a fin inscripciones procedentes de la Descripción de la provincia Bethica, redactada en 1570 y dirigida a Joachim Hopper (1523-1576), presidente del consejo de Flandes; de ella conocemos una única copia, no autógrafa, en la Biblioteca Marciana de Venecia, que ha pasado desapercibida hasta hace relativamente poco y que continúa a día de hoy sin estudiar. ${ }^{23}$ Agustín copió las inscripciones de la obra de inicio a fin, omitiendo también algunos textos pero manteniendo el mismo orden que en el original. ${ }^{24}$ Sabemos que la obra de Franco tuvo una cierta difusión entre los humanistas del norte de Europa: Hopper se la dio a Augerius Busbequius, y este le transmitió los epígrafes a Pighius solo un año después de su redacción, en $1571 .{ }^{25}$ Con todo, parece más verosímil suponer que el libro llegase a Agustín procedente del autor y no de Hopper, al igual que la Suma, y muy

18 Suma de las inscripciones romanas y memorias de la Bética. Sevilla, Biblioteca Capitular y Colombina, ms. 84-8-37 (original autógrafo).

19 Monumento de inscripciones romanas de varias piedras de pueblos de la Andalucía y España. Milán, Biblioteca Braidense, ms. AH X 29 (original autógrafo).

20 Descripción de la provincia Bethica nombrada Andaluzia con muchos monumentos e inscripciones antiguas que del tiempo de Romanos en ella se hallan dirigida al muy illustre señor Ioachimo Opero, presidente del Real Consejo de Flandes. Venecia, Biblioteca Nazionale Marciana, ms. Lat. X, 258 (3852) (copia).

21 Compendio y breve summario de la descripción de la provincia Bethica / nombrada Andaluzia [y] la antigüedad e [historia] no/table de la celebrada [villa?] / de Estepa con algunos / titulos e inscriptiones [...] / [...] se hallan del / tiempo de Romanos / dirigida al / [sr. Iofredo Lescaro?] / gobernador del marquesado /de Estepa. Madrid, Biblioteca Nacional de España, ms. 1834 (original autógrafo).

22 Esta era la única fuente de los Adversaria que se había dado a conocer hasta ahora; uid. Carbonell - Gimeno 2016.

23 Vid. nota 20. Después de una breve mención por parte de Hübner en el Supplementum (p. LXXXI) al CIL II, no hay ninguna mención del manuscrito hasta González Germain 2011, 75.

24 Las dos únicas excepciones son, significativamente, una inscripción tarraconense (CIL II 4165= II²/14, 1036), transcrita en la obra de Franco en tamaño más pequeño y sin un marco epigráfico, y una leyenda parcial de una moneda de Italica.

25 Berlín, Staatsbibliothek, ms. Lat. fol. 61, ff. 197r-v (Ex libro de antiquitatibus Baeticae manuscripto quem Hopperus Augerio misit) y 199v-200 (Ex libro quem Hopperus Augerio misit ex Hispania Io. Franci de Baeticae antiquitatibus). 
probablemente con posterioridad a esta. No podemos determinar a ciencia cierta cuál de los dos es el «libro d'inscritioni della Bethica [...] assai ridiculo», aunque la fecha de elaboración de la Descripción es más cercana a la fecha de la referencia, así como mayor es el número de falsos que contiene, tema al cual Agustín era muy sensible. ${ }^{26}$

Una vez más, a pesar del poco aprecio hacia el trabajo de uno de sus contemporáneos hispánicos que muestran las palabras del obispo, tenemos evidencias claras de que se sirvió de al menos dos de sus trabajos, aunque fuera de manera privada y sin hacerlo constar.

\section{Otras fuentes utilizadas por Agustín}

El Liber Parmensis, del que hemos hablado ya con relación a la serie tarraconense, no solo sirvió para completar la síloge de Pons d'Icart, sino que Agustín copió buena parte de su contenido en los ff. $82 \mathrm{r}$ y $83 \mathrm{r}-\mathrm{v}$, excluyendo aquí los textos de Tarragona y sin dar ninguna indicación acerca de su fuente. Del mismo modo, todas las inscripciones del f. 84r, que como hemos dicho presentaba un formato distinto de los demás, al estar doblado tanto transversal como longitudinalmente, proceden del Liber Parmensis. Entre los epígrafes copiados, no solo figuran aquellos que formaban parte de la síloge original del primer cuarto del s. XVI, sino que encontramos también aquellos que fueron añadidos por las otras tres manos que intervienen en el códice, que recientemente se han datado a mediados del s. XVI por su paleografía, y para las que ahora podemos establecer la redacción de los Aduersaria como terminus ante quem. ${ }^{27}$

Todavía hemos podido identificar otro manuscrito epigráfico utilizado con seguridad por Agustín. Se trata del ms. 5973 de la BNE (al que Hübner apodó incorrectamente Docampo), del que Agustín copió 49 textos desde el f. 74v col. I hasta el f. 75v col. I. ${ }^{28}$ Se trata de un manuscrito sin firmar escrito por una mano hispana, y que incluye inscripciones modernas hasta 1562 (f. 3r); podemos suponer que el códice fue completado en los años inmediatamente sucesivos a esta fecha, antes de que Agustín lo consultara en la década siguiente. Como con las demás fuentes, Agustín vació los epígrafes del códice sin indicar su proveniencia, omitiendo no pocos textos, pero siempre en sentido descendente, lo que permite identificar la fuente sin demasiadas dificultades. Otro signo inequívoco son las cuatro inscripciones de Tivoli que Agustín copia (presuntamente por error), de entre las numerosas inscripciones itálicas de la fuente original. ${ }^{29}$ Por último, es interesante subrayar que el manuscrito contenía no pocas inscripciones falsas, que Agustín deja de copiar en algunos casos al cabo de unas pocas palabras, sin duda por considerarlas espurias. ${ }^{30}$

26 La detección y referencias a ejemplares falsos tanto numismáticos como epigráficos puebla todo su epistolario a partir de inicios de la década de 1550. Recuérdese solamente que el arzobispo titula el capítulo XI de sus Dialogos de medallas, inscriciones y otras antigüedades, Tarragona, Felipe Mey, 1587, «De las medallas falsas y letreros falsos y de los que han escrito de medallas y inscriciones» (443-470).

27 Se trata de $C I L$ II $2200=$ II $^{2} / 7,258$ y $C I L$ II $2276=$ II $^{2} / 7,459$, CIL II 3033 y $C I L$ II 2559, correspondientes respectivamente a las manus b, $c$ y $d$ del Liber Parmensis; cf. González Germain 2016, pp. 36-42.

28 Madrid, Biblioteca Nacional de España, ms. 5973. Existe un artículo monográfico con errores de peso: Louzado 1998; véase igualmente Hernando Sobrino 2009, 237-240, y González Germain 2011, 71 y 132-133.

29 En concreto, CIL XIV 3643, 3863, 3661 y 3543 (f. 75r-v).

30 Este es el caso de CIL II $83^{*}, 80^{*}$ y $85^{*}$ (f. $\left.74 \mathrm{v}\right)$. 
Más allá de las seis fuentes epigráficas vaciadas por Agustín que hemos identificado en este artículo, quedan todavía tres series menores por identificar. Quizás la más homogénea sea la serie saguntina que ocupa parte de los ff. 75v, 76r y 76v. Está compuesta por un conjunto de siete epígrafes copiados por Agustín y completado por otro de veinticuatro debido a la mano de Martín López de Bailo, sin que se pueda determinar si el añadido de Bailo es o no contemporáneo a las copias de Agustín.

En segundo lugar, en el f. 75v, cuando Agustín acaba con la copia de los ejemplares del manuscrito 5973 de la Biblioteca Nacional, inicia un conjunto que contiene epígrafes de Granada (1), Córdoba (1), Montoro (1), Arjona (1) Baeza (1) y Vila Viçosa (17). En cuanto a estos últimos, la colación con la obra de Resende y con otras síloges coetáneas no ha sido fructífera. Así podemos afirmar que la fuente manuscrita que sin duda subyace se encuentra perdida en la actualidad.

Finalmente, en el f. 72r-v encontramos una serie de inscripciones de la Bética, algunas de las cuales Hübner atribuyó a la tradición de Fernández Franco. Son de Castro del Río (6), Porcuna (2), Espejo (1), Jerez [de hecho, Cádiz] (1), Osuna (2), Archidona (1) y Málaga (1). Sin tener pruebas concluyentes, la hipótesis de Hübner no resulta descabellada, aunque de ser así se trataría de una obra (o síloge epigráfica) distinta de las conocidas hasta la fecha.

\section{Conclusiones}

A partir de las noticias extraídas del epistolario sabemos, pues, que el arzobispo habría podido empezar a copiar el libro de Icart hacia mediados de 1576, junto a una de las obras de Fernández Franco, si no las dos; a la misma época pertenecería la copia de la síloge de Joan Armengol y el misterioso Bux., que interrumpen la serie de Icart. Finalmente, si nos atenemos a la homogeneidad de la caligrafía de todo el conjunto, suponemos que la copia de todo él debe situarse alrededor de los primeros años del segundo lustro de la década de los setenta. ${ }^{31}$

Sabemos ahora que el humanista había acumulado una cantidad considerable de manuscritos epigráficos y lo imaginamos trabajando con distintos a la vez (la obra de Icart, la Suma y la Descripción de Fernández Franco, la síloge de Armengol, el Liber Parmensis o el BNE ms. 5973) con el objetivo de elaborar su propia síloge de inscripciones hispanas, la cual, de hecho, es quizás la más voluminosa de cuantas se han conservado de la época. Esta es la razón por la cual el CIL otorgó al arzobispo una importancia que, a la vista de lo expuesto, debe relativizarse. Agustín se limita a reproducir o emendar ex cathedra la documentación que se le suministra, en muchos casos a pesar de la opinión que le merecen sus suministradores.

Es evidente que el personaje era considerado una autoridad en cuestiones anticuarias y sobre todo en aquellas relacionadas con la epigrafía y la numismática, de ahí que fuera requerido su papel de censor por parte de aquellos que querían obtener un plácet fuera de toda discusión; así lo formula Pons d'Icart en su misiva. No olvidemos además el valor añadido que suponía contar con una imprenta bajo su tutela.

Sin embargo, no nos encontramos ante un arqueólogo o interesado por el arte anticuario. Agustín es un verdadero filólogo; contempla el texto epigráfico como si

31 Quizás no sea casualidad haber comenzado la copia de títulos de Tarragona al conocer su promoción a dicha sede arzobispal a principios de marzo de 1576. uid. Carbonell 1997, 1330-1333. 
se tratara de una fuente literaria. Su máximo interés reside en comprender el texto mismo de los epígrafes; no existen casi nunca noticias sobre los soportes o las ubicaciones o el contexto arqueológico de los hallazgos. Sí, en cambio, se observa escrupulosidad en mantener la disposición en líneas, en comprender las abreviaturas o en realizar correcciones casi a bote pronto, según discurre la copia. Sin duda la pericia, adquirida en largas conversaciones con sus añorados colegas romanos, le permitía proponer lecciones más creíbles que las que a veces leía o eliminar epígrafes claramente falsos o recentiores. Fue un experto conocedor y consultor sobre cuestiones epigráficas, como lo atestiguan sus constantes referencias y discusiones epistolares a lo largo de su vida, aunque su interés por la numismática superó al de la epigrafía. De ello es testimonio el valor que atribuye a la documentación numismática en su última publicación póstuma, los Dialogos de medallas (Tarragona 1587).

\section{Apéndice. Tabla comparativa de los Aduersaria de Antonio Agustín y sus fuentes}

A continuación ofrecemos la relación de inscripciones de los ff. $72 \mathrm{r}-2 \mathrm{v}$ y $84 \mathrm{r}$ del ms. 5781, agrupadas según la fuente de procedencia, con la referencia de cada epígrafe en la fuente original y en la síloge de Agustín (para la que señalamos el número de folio y la columna en la que figura cada inscripción). Indicamos la localización de los textos según Agustín y, en su caso, su correcta correspondencia entre corchetes.

Inscripciones de Fernández Franco, Suma (Sevilla, Biblioteca Colombina, ms. 84-8-37)

\begin{tabular}{|l|l|l|l|}
\hline${\text { CIL II }=\mathrm{II}^{2}}$ & Lugar & Suma & Agustín \\
\hline $2246=7,390$ & Córdoba & 4 & $73 \mathrm{r}(\mathrm{II})$ \\
\hline 4701 & Córdoba & $4 \mathrm{v}$ & $73 \mathrm{r}(\mathrm{II})$ \\
\hline 4712 & Córdoba & 5 & $73 \mathrm{r}(\mathrm{II})$ \\
\hline $2225=7,284$ & Córdoba & $5 \mathrm{v}$ & $73 \mathrm{r}(\mathrm{II})$ \\
\hline $2281=7,470$ & Córdoba & 6 & $73 \mathrm{r}(\mathrm{II})$ \\
\hline $2300=7,517$ & Córdoba & $6 \mathrm{v}$ & $73 \mathrm{r}(\mathrm{II})$ \\
\hline 4719 & Córdoba & 7 & $73 \mathrm{r}(\mathrm{II})$ \\
\hline $2301=7,518$ & Córdoba & $7 \mathrm{v}$ & $73 \mathrm{r}(\mathrm{II})$ \\
\hline $139 \mathrm{a} *=5,12 *$ & Montemayor & $8 \mathrm{r}$ & $73 \mathrm{r}(\mathrm{II})$ \\
\hline $1534=5,495$ & Montemayor & $8 \mathrm{v}$ & $73 \mathrm{r}(\mathrm{II})$ \\
\hline $1536=5,498$ & Montemayor & 9 & $73 \mathrm{v}(\mathrm{I})$ \\
\hline $1529=5,490$ & Montemayor & $9 \mathrm{v}$ & $73 \mathrm{v}(\mathrm{I})$ \\
\hline $1527=5,488$ & Montemayor & 10 & $73 \mathrm{v}(\mathrm{I})$ \\
\hline $1528=5,489$ & Montemayor & $10 \mathrm{v}$ & $73 \mathrm{v}(\mathrm{I})$ \\
\hline $1530=5,491$ & Montemayor & $10 \mathrm{v}$ & $73 \mathrm{v}(\mathrm{I})$ \\
\hline $2188=7,197$ & Pedro Abad & 11 & $73 \mathrm{v}(\mathrm{I})$ \\
\hline
\end{tabular}




\begin{tabular}{|c|c|c|c|}
\hline $2187=7,212$ & El Carpio & 12 & $73 v(I)$ \\
\hline $2156=7,139$ & Montoro & $12 \mathrm{v}$ & $73 v(I)$ \\
\hline $2163=7,143$ & Montoro & 13 & $73 \mathrm{v}(\mathrm{I})$ \\
\hline $2162=7,147$ & Montoro & $13 \mathrm{v}$ & $73 \mathrm{v}(\mathrm{I})$ \\
\hline $2165=7,148$ & Montoro & $14 \mathrm{v}$ & $73 \mathrm{v}(\mathrm{I})$ \\
\hline $2166=7,155$ & Montoro & 15 & $73 \mathrm{v}(\mathrm{I})$ \\
\hline $2176=7,176$ & Montoro & $15 \mathrm{v}$ & $73 \mathrm{v}(\mathrm{I})$ \\
\hline $2171=7,165$ & Montoro & $15 \mathrm{v}$ & $73 \mathrm{v}(\mathrm{I})$ \\
\hline $2126=7,93$ & Porcuna & 17 & $73 \mathrm{v}(\mathrm{I})$ \\
\hline $2146=7,116$ & Porcuna & $17 \mathrm{v}$ & $73 \mathrm{v}$ (II) \\
\hline $2131=7,98$ & Porcuna & $18 \mathrm{r}$ & $72 \mathrm{v}(\mathrm{I})$ \\
\hline $2149=7,119$ & Porcuna & $18 \mathrm{r}$ & $\begin{array}{l}72 \mathrm{v}(\mathrm{I}) \\
73 \mathrm{v}(\mathrm{I})\end{array}$ \\
\hline $2132=7,100$ & Porcuna & $18 \mathrm{v}$ & $72 \mathrm{v}(\mathrm{I})$ \\
\hline $2136=7,101$ & Porcuna & $19 \mathrm{r}$ & $73 \mathrm{v}(\mathrm{II})$ \\
\hline $2127=7,92$ & Porcuna & $19 r$ & $73 \mathrm{v}$ (II) \\
\hline $2135=7,127$ & Porcuna & $19 \mathrm{v}$ & $72 \mathrm{v}(\mathrm{I})$ \\
\hline $2144=7,114$ & Porcuna & $20 r$ & $73 \mathrm{v}$ (II) \\
\hline $2143=7,113$ & Porcuna & $20 r$ & $73 \mathrm{v}$ (II) \\
\hline $2106=7,69$ & Arjona & $21 r$ & $73 \mathrm{v}$ (II) \\
\hline $2109=7,71$ & Arjona & $21 \mathrm{v}$ & $73 \mathrm{v}$ (II) \\
\hline $2108=7,73$ & Arjona & $22 r$ & $73 \mathrm{v}$ (II) \\
\hline $2105=7,68$ & Arjona & $22 v$ & $73 \mathrm{v}$ (II) \\
\hline $1686=5,157$ & Torredonjimeno & $23 r$ & $73 \mathrm{v}(\mathrm{II})$ \\
\hline $1703=5,129$ & Martos & $23 r$ & $73 \mathrm{v}(\mathrm{II})$ \\
\hline $1680=5,88$ & Martos & $23 \mathrm{v}$ & $73 \mathrm{v}(\mathrm{II})$ \\
\hline $1660=5,65$ & Martos & $24 r$ & $73 \mathrm{r}(\mathrm{I})$ \\
\hline $1692=5,103$ & Martos & $24 \mathrm{v}$ & $73 \mathrm{r}(\mathrm{I})$ \\
\hline $1694=5,102$ & Martos & $24 \mathrm{v}$ & $73 \mathrm{r}(\mathrm{I})$ \\
\hline $1679=5,87$ & Martos & $25 r$ & $73 r(I)$ \\
\hline $1704=5,119$ & Martos & $25 \mathrm{vr}$ & 73r (I) \\
\hline $1678=5,89$ & Martos & $26 r$ & $73 \mathrm{r}(\mathrm{I})$ \\
\hline $1663=5,69$ & Martos & $26 \mathrm{v}$ & $73 \mathrm{r}(\mathrm{I})$ \\
\hline $1670=5,75$ & Martos & $28 \mathrm{v}$ & $73 \mathrm{r}(\mathrm{I})$ \\
\hline 3289 & Cazlona & $30 \mathrm{v}$ & $73 \mathrm{r}(\mathrm{I})$ \\
\hline 3297 & Cazlona & $31 \mathrm{r}$ & $73 \mathrm{r}(\mathrm{I})$ \\
\hline 3310 & Cazlona & $31 v$ & $74 \mathrm{r}(\mathrm{II})$ \\
\hline
\end{tabular}




\begin{tabular}{|c|c|c|c|}
\hline $3269 \mathrm{a}$ & Cazlona & $32 r$ & $74 \mathrm{r}$ (II) \\
\hline $3363=5,32$ & Jaén & $32 v$ & 74r (II) \\
\hline $3367=5,35$ & Jaén & $32 v$ & $74 \mathrm{r}$ (II) \\
\hline $1721=5,232$ & Alcaudete & $33 r$ & $74 \mathrm{r}(\mathrm{II})$ \\
\hline $1519=5,583$ & Aguilar de la Frontera & $33 r$ & $74 \mathrm{r}(\mathrm{II})$ \\
\hline $1423=5,871$ & Cerro de Sabora & $34 \mathrm{r}$ & $74 \mathrm{r}(\mathrm{II})$ \\
\hline $2015=5,783$ & Cerro del Castillón & $35 r$ & $72 \mathrm{v}(\mathrm{I})^{32}$ \\
\hline $2023=5,791$ & Cerro del Castillón & $35 \mathrm{v}$ & $72 \mathrm{v}(\mathrm{I})$ \\
\hline $2022=5,790$ & Cerro del Castillón & $36 r$ & $72 \mathrm{v}(\mathrm{I})$ \\
\hline $2024=5,805$ & Cerro del Castillón & $36 \mathrm{r}$ & $72 \mathrm{v}(\mathrm{I})$ \\
\hline $2034=5,742$ & Antequera & $36 v$ & $72 \mathrm{v}(\mathrm{I})$ \\
\hline $2006=5,838$ & Sierra de Abdalagis & $37 \mathrm{r}$ & $72 \mathrm{v}(\mathrm{I})$ \\
\hline $2005=5,837$ & Sierra de Abdalagis & $37 \mathrm{v}$ & $72 \mathrm{v}(\mathrm{I})$ \\
\hline 4690 & Málaga & $38 \mathrm{v}$ & $72 \mathrm{v}(\mathrm{I})$ \\
\hline 1977 & Málaga & $39 v$ & $72 \mathrm{r}(\mathrm{I})$ \\
\hline 1967 & Málaga & $40 \mathrm{r}$ & $72 \mathrm{v}(\mathrm{II})$ \\
\hline 1966 & Málaga & $40 v$ & $72 \mathrm{r}(\mathrm{I})$ \\
\hline 1968 & Málaga & $41 \mathrm{r}$ & $72 \mathrm{r}(\mathrm{I})$ \\
\hline $2071=5,622$ & Granada & $41 \mathrm{v}$ & $72 \mathrm{v}(\mathrm{II})$ \\
\hline $2087=5,637$ & Granada & $42 r$ & $72 \mathrm{v}(\mathrm{II})$ \\
\hline $2064=5,681$ & Cerro de los Infantes & $42 v$ & $72 \mathrm{r}(\mathrm{I})$ \\
\hline 1222 & Sevilla & $43 v$ & $72 \mathrm{r}(\mathrm{I})$ \\
\hline 1197 & Sevilla & $44 \mathrm{r}$ & $72 \mathrm{v}$ (II) \\
\hline 1324 & Medina Sidonia & $45 r$ & $72 \mathrm{v}(\mathrm{II})$ \\
\hline 1296 & Lebrija & $45 \mathrm{v}$ & $72 \mathrm{r}(\mathrm{I})$ \\
\hline $1425=5,854$ & Cortijo del Tajo & $47 \mathrm{r}$ & $72 \mathrm{r}(\mathrm{I})$ \\
\hline $1424=5,853$ & Cortijo del Tajo & $47 \mathrm{v}$ & $72 \mathrm{r}(\mathrm{I})$ \\
\hline $1416=5,1065$ & Osuna & $48 v$ & $72 \mathrm{r}(\mathrm{I})$ \\
\hline $278 d^{*}$ & Guisando & $49 \mathrm{r}$ & $72 \mathrm{r}(\mathrm{I})$ \\
\hline $278 b^{*}$ & Guisando & $49 v$ & $72 \mathrm{r}(\mathrm{I})$ \\
\hline $278 a^{*}$ & Guisando & $49 v$ & $72 \mathrm{r}(\mathrm{I})$ \\
\hline 3052 & Guisando & $50 \mathrm{r}$ & $72 \mathrm{r}(\mathrm{I})$ \\
\hline $278 \mathrm{c} *$ & Guisando & $50 \mathrm{r}$ & $72 \mathrm{r}(\mathrm{I})$ \\
\hline
\end{tabular}

32 Nótese que a partir de este momento Agustín no sigue estrictamente la secuencia que presenta la Suma en el folio que esperaríamos; contrariamente, la secuencia de Fdez. Franco 'retrocede' a los ff. 72v-72r, donde sí se aprecia la continuidad. 
Inscripciones del ms. 5973 de la BNE

\begin{tabular}{|c|c|c|c|}
\hline $\mathrm{CIL} \mathrm{II}=\mathrm{II}^{2}$ & Lugar & ms. 5973 & Agustín \\
\hline IHC 84 & Lebrija & $2 \mathrm{v}$ & $74 \mathrm{v}(\mathrm{I})$ \\
\hline $\begin{array}{l}\text { moderna (Diva } \\
\text { Blanca...) }\end{array}$ & Jerez de la Frontera & $8 v$ & $74 \mathrm{v}(\mathrm{I})$ \\
\hline $2099=5,296$ & Lucena [Zambra] & $20 v$ & $74 \mathrm{v}(\mathrm{I})$ \\
\hline $2098=5,294$ & Cabra [Zambra] & $20 v$ & $74 \mathrm{v}(\mathrm{I})$ \\
\hline 3180 & Valeria & $22 \mathrm{r}$ & $74 \mathrm{v}(\mathrm{I})$ \\
\hline 1235 & Sevilla & $23 r$ & $74 \mathrm{v}(\mathrm{I})$ \\
\hline 1047 & Constantina & $23 \mathrm{v}$ & $74 \mathrm{v}(\mathrm{I})$ \\
\hline 1046 & Constantina & $23 \mathrm{v}$ & $74 \mathrm{v}(\mathrm{I})$ \\
\hline 759 & Alcántara & $26 v$ & $74 \mathrm{v}(\mathrm{I})$ \\
\hline 760 & Alcántara & $26 \mathrm{v}$ & $74 \mathrm{v}(\mathrm{I})$ \\
\hline 761 & Alcántara & $27 \mathrm{r}$ & $74 \mathrm{v}$ (II) \\
\hline $2062=5,677$ & Pinos Puente & $32 \mathrm{v}$ & $74 \mathrm{v}$ (II) \\
\hline $2064=5,681$ & Pinos Puente & $32 v$ & $74 \mathrm{v}$ (II) \\
\hline $83^{*}$ & Capera & $37 \mathrm{r}$ & 74v (II) \\
\hline $81 *$ & Capera & $38 \mathrm{r}$ & $74 \mathrm{v}$ (II) \\
\hline 825 & Capera & $39 r$ & $74 \mathrm{v}(\mathrm{II})$ \\
\hline $80^{*}$ & Capera & $39 v$ & $74 \mathrm{v}$ (II) \\
\hline $85^{*}$ & Capera & $40 v-41 r$ & $74 \mathrm{v}$ (II) \\
\hline 4657 & Vía de la Plata & $41 \mathrm{v}$ & $74 \mathrm{v}$ (II) \\
\hline $231 *$ & Vía de la Plata & $41 \mathrm{v}$ & $74 \mathrm{v}$ (II) \\
\hline 813 & Capera & $42 r$ & $74 \mathrm{v}$ (II) \\
\hline 1969 & Málaga & $45 \mathrm{v}$ & $74 \mathrm{v}$ (II) \\
\hline 1966 & Málaga & $45 v$ & $74 \mathrm{r}(\mathrm{I})$ \\
\hline 1973 & Málaga & $46 r$ & $74 \mathrm{r}(\mathrm{I})$ \\
\hline $84 *$ & Capera & $48 \mathrm{r}$ & $74 \mathrm{r}(\mathrm{I})$ \\
\hline $15^{*}$ & Évora & $56 \mathrm{r}$ & $74 \mathrm{r}(\mathrm{I})$ \\
\hline 1200 & Sevilla & $59 \mathrm{r}$ & $74 \mathrm{r}(\mathrm{I})$ \\
\hline $1473=5,1164$ & Écija & $59 \mathrm{v}$ & $74 \mathrm{r}(\mathrm{I})$ \\
\hline $1474=5,1165$ & Écija & $59 \mathrm{v}$ & $74 \mathrm{r}(\mathrm{I})$ \\
\hline $2021=5,798$ & Antequera & $62 \mathrm{r}$ & $74 \mathrm{r}(\mathrm{I})$ \\
\hline $2042=5,848$ & Antequera & $62 \mathrm{v}$ & $74 \mathrm{r}(\mathrm{I})$ \\
\hline $2029=5,780$ & Antequera & $70 v$ & $74 \mathrm{r}(\mathrm{I})$ \\
\hline $2010=5,846$ & Antequera & $71 \mathrm{v}$ & $74 \mathrm{r}(\mathrm{I})$ \\
\hline
\end{tabular}




\begin{tabular}{|l|l|l|l|}
\hline 2329 & Peñaflor & $110 \mathrm{r}$ & $74 \mathrm{r}(\mathrm{I})$ \\
\hline 2330 & Peñaflor & $110 \mathrm{r}$ & $74 \mathrm{r}(\mathrm{I})$ \\
\hline 1185 & Sevilla & $113 \mathrm{r}$ & $75 \mathrm{r}(\mathrm{II})$ \\
\hline 1169 & Sevilla & $114 \mathrm{r}$ & $75 \mathrm{r}(\mathrm{II})$ \\
\hline 1953 & Cártama & $114 \mathrm{v}$ & $75 \mathrm{r}(\mathrm{II})$ \\
\hline 1956 & Cártama & $115 \mathrm{r}-\mathrm{v}$ & $75 \mathrm{r}(\mathrm{II})$ \\
\hline $2048=5,758$ & Antequera & $116 \mathrm{r}$ & $75 \mathrm{r}(\mathrm{II})$ \\
\hline $2046=5,756$ & Antequera & $116 \mathrm{r}$ & $75 \mathrm{r}(\mathrm{II})$ \\
\hline 1945 & Álora & $116 \mathrm{v}$ & $75 \mathrm{r}(\mathrm{II})$ \\
\hline XIV 3643 & Tivoli & $117 \mathrm{r}$ & $75 \mathrm{r}(\mathrm{II})$ \\
\hline $1650=5,256$ & Carcabuey & $131 \mathrm{v}$ & $75 \mathrm{r}(\mathrm{II})$ \\
\hline 3278 & Cazlona & $134 \mathrm{v}$ & $75 \mathrm{r}(\mathrm{II})$ \\
\hline XIV 3863 & Tivoli & $146 \mathrm{r}$ & $75 \mathrm{r}(\mathrm{II})$ \\
\hline XIV 3661 & Tivoli & $146 \mathrm{v}$ & $75 \mathrm{v}(\mathrm{I})$ \\
\hline XIV 3543 & Tivoli & $146 \mathrm{v}$ & $75 \mathrm{v}(\mathrm{I})$ \\
\hline IHC 54 & Oliva de Plasencia & $171 \mathrm{v}$ & $75 \mathrm{v}(\mathrm{I})$ \\
\hline
\end{tabular}

Inscripciones no tarraconenses del Liber Parmensis (Parma, Bibl. Palatina, Z II 22763)

\begin{tabular}{|l|l|l|l|}
\hline CIL II $=\mathrm{II}^{2}$ & Lugar & Liber Parm. & Agustín \\
\hline 4691 & Málaga & 10 & $75 \mathrm{r}(\mathrm{I})$ \\
\hline 1975 & Málaga & $10 \mathrm{v}$ & $75 \mathrm{r}(\mathrm{I})$ \\
\hline 1956 & Cártama & $11 \mathrm{r}$ & $75 \mathrm{r}(\mathrm{I})$ \\
\hline 1957 & Cártama & $11 \mathrm{r}$ & $75 \mathrm{r}(\mathrm{I})$ \\
\hline 1947 & Alora & $11 \mathrm{r}$ & $75 \mathrm{r}(\mathrm{I})$ \\
\hline $1403=5,1023$ & Osuna & $11 \mathrm{v}$ & $75 \mathrm{r}(\mathrm{I})$ \\
\hline $1416=5,1065$ & Osuna & $11 \mathrm{v}$ & $75 \mathrm{r}(\mathrm{I})$ \\
\hline $1474=5,1165$ & Écija & $11 \mathrm{v}$ & $75 \mathrm{r}(\mathrm{I})$ \\
\hline $1490=5,1200$ & Écija & $11 \mathrm{v}$ & $75 \mathrm{r}(\mathrm{I})$ \\
\hline 4512 & Barcelona & $9 \mathrm{v}$ & $76 \mathrm{r}(\mathrm{II})$ \\
\hline 4528 & Barcelona [Vilassar de Mar] & $9 \mathrm{v}$ & $76 \mathrm{r}(\mathrm{II})$ \\
\hline 3555 & Elche & $10 \mathrm{r}$ & $76 \mathrm{r}(\mathrm{II})$ \\
\hline 3567 & Elche & $10 \mathrm{r}$ & $76 \mathrm{r}(\mathrm{II})$ \\
\hline 3626 & Játiva & $10 \mathrm{r}$ & $76 \mathrm{r}(\mathrm{II})$ \\
\hline 1965 & Denia & $10 \mathrm{r}$ & $76 \mathrm{r}(\mathrm{II})$ \\
\hline 3660 & Ibiza & $10 \mathrm{r}$ & $76 \mathrm{r}(\mathrm{II})$ \\
\hline $164 *$ & Denia & $11 \mathrm{v}$ & $76 \mathrm{r}(\mathrm{II})$ \\
\hline $1475=5,1171$ & Écija & $11 \mathrm{v}$ & $76 \mathrm{v}(\mathrm{I})$ \\
\hline
\end{tabular}




\begin{tabular}{|c|c|c|c|}
\hline $1488=5,1195$ & Écija & $12 r$ & $76 \mathrm{v}(\mathrm{I})$ \\
\hline $1511=5,1254$ & Écija & $12 \mathrm{r}$ & 76v (I) \\
\hline $1501=5,1224$ & Écija & $12 \mathrm{r}$ & $76 \mathrm{v}(\mathrm{I})$ \\
\hline $2243=7,347$ & Córdoba & $12 \mathrm{v}$ & 76v (I) \\
\hline $2201=7,260$ & Córdoba & $12 \mathrm{v}$ & $76 \mathrm{v}(\mathrm{I})$ \\
\hline $2202=7,262$ & Córdoba & $12 \mathrm{v}$ & $76 \mathrm{v}(\mathrm{I})$ \\
\hline $1537=5,499$ & Montemayor & $13 r$ & $76 \mathrm{v}(\mathrm{I})$ \\
\hline $356^{*}$ & Murcia & $13 r$ & 76v (II) \\
\hline $3856=14,354$ & Sagunto & $13 \mathrm{v}$ & 76v (II) \\
\hline $3914=14,479$ & Sagunto & $13 \mathrm{v}$ & $76 \mathrm{v}$ (II) \\
\hline $3877=14,380$ & Sagunto & $13 \mathrm{v}$ & 76v (II) \\
\hline $3913=14,476$ & Sagunto & $13 \mathrm{v}$ & 76v (II) \\
\hline $3896=14,644$ & Sagunto & $13 \mathrm{v}$ & $76 \mathrm{v}$ (II) \\
\hline $3940=14,533$ & Sagunto & $14 \mathrm{r}$ & 76v (II) \\
\hline $3876=14,625$ & Sagunto & $14 \mathrm{r}$ & 76v (II) $i . m$ \\
\hline $363^{*}$ & Denia & $14 \mathrm{r}$ & 76v (II) \\
\hline 3605 & Gandía & $14 \mathrm{r}$ & 76r (I) \\
\hline 3602 & Gandía & $14 \mathrm{r}$ & 76r (I) \\
\hline 3616 & Gandía & $14 \mathrm{v}$ & 76r (I) \\
\hline 761 & Alcántara & $14 \mathrm{v}$ & 76r (I) \\
\hline 759 & Alcántara & $14 \mathrm{v}$ & 76r (I) \\
\hline 3033 & Alcalá de Henares & $1 \mathrm{r}[$ mano c $]$ & $84 \mathrm{r}$ \\
\hline 2559 & La Coruña & $4 \mathrm{r}[$ mano d] & $84 \mathrm{r}$ \\
\hline $2200=7,258$ & Córdoba & $4 \mathrm{v}[$ mano b] & $84 \mathrm{r}$ \\
\hline $2276=7,459$ & Córdoba & $4 \mathrm{v}[$ mano $\mathrm{b}]$ & $84 \mathrm{r}$ \\
\hline 4718 & Córdoba & $4 \mathrm{v}[$ mano b] & $84 \mathrm{r}$ \\
\hline 3034 & Alcalá de Henares & 5r [mano b] & $84 \mathrm{r}$ \\
\hline 4458 & Isona & $16 \mathrm{v}$ & $84 \mathrm{r}$ \\
\hline 4464 & Isona & $1 \mathrm{r}$ & $84 \mathrm{r}$ \\
\hline 4473 & Isona & $16 \mathrm{v}$ & $84 \mathrm{r}$ \\
\hline 4468 & Isona & $16 \mathrm{v}$ & $84 \mathrm{r}$ \\
\hline 4472 & Isona & $16 \mathrm{v}$ & $84 \mathrm{r}$ \\
\hline 4465 & Isona & $16 \mathrm{v}$ & $84 \mathrm{r}$ \\
\hline
\end{tabular}


Inscripciones de Fernández Franco, Descripción (Bibl. Marciana, ms. Lat. X, 258) ${ }^{33}$

\begin{tabular}{|c|c|c|c|}
\hline $\mathrm{CIL} \mathrm{II} \mathrm{=} \mathrm{II}^{2}$ & Lugar & Descripción & Agustín \\
\hline $2366=7,853$ & Cerro del Cabezo & 33 & $77 \mathrm{r}(\mathrm{I})$ \\
\hline 859 & Ledesma & 34 & $77 \mathrm{r}(\mathrm{I})$ \\
\hline $2349=7,776$ & Solia & 35 & $77 \mathrm{r}(\mathrm{I})$ \\
\hline $2154=5,430$ & Cañete de las Torres & 43 & $77 \mathrm{r}(\mathrm{I})$ \\
\hline $2226=7,311$ & Córdoba & 52 & $77 \mathrm{r}(\mathrm{I})$ \\
\hline $2200=7,238$ & Córdoba & 53 & $77 \mathrm{r}(\mathrm{I})$ \\
\hline 4718 & Córdoba & 55 & $77 \mathrm{r}(\mathrm{I})$ \\
\hline $2232=7,246$ & Córdoba & 57 & $77 \mathrm{r}(\mathrm{I})$ \\
\hline 4700 & Montoro & 73 & $77 \mathrm{r}(\mathrm{I})$ \\
\hline $1532=5,492$ & Montemayor & 92 & $77 \mathrm{v}(\mathrm{I})$ \\
\hline $145^{*}=5,11^{*}$ & Espejo & 95 & $77 \mathrm{v}(\mathrm{I})$ \\
\hline $1553=5,440$ & Espejo & 96 & $77 \mathrm{v}(\mathrm{I})$ \\
\hline $1554=5,442$ & Espejo & 98 & $77 \mathrm{v}(\mathrm{I})$ \\
\hline $2189=7,214$ & El Carpio & 105 & $77 \mathrm{v}(\mathrm{I})$ \\
\hline $1036=7,975$ & Casas de Reina & 108 & $77 \mathrm{v}(\mathrm{I})$ \\
\hline $1471=5,1162$ & Écija & 109 & $77 \mathrm{v}(\mathrm{I})$ \\
\hline $1673=5,80$ & Martos & 113 & $77 \mathrm{v}(\mathrm{I})$ \\
\hline $2131=7,98$ & Porcuna & 119 & $77 \mathrm{v}(\mathrm{I})$ \\
\hline 1733 & Cádiz & 129 & $<77 \mathrm{v}(\mathrm{I})>^{33}$ \\
\hline 1792 & Cádiz & 129 & $77 \mathrm{v}$ (II) \\
\hline 1170 & Sevilla & 135 & $77 \mathrm{v}(\mathrm{II})$ \\
\hline 1165 & Sevilla & 138 & $77 \mathrm{v}(\mathrm{II})$ \\
\hline $74 *$ & Lebrija & 140 & $77 \mathrm{v}(\mathrm{II})$ \\
\hline $99 *$ & Morón de la Frontera & 142 & 77v (II) \\
\hline 1309 & Jerez de la Frontera & 143 & $77 \mathrm{v}$ (II) \\
\hline $127^{*}$ & Carmona & 144 & $77 \mathrm{v}(\mathrm{II})$ \\
\hline $2038=5,748$ & Antequera & 147 & $77 \mathrm{v}$ (II) \\
\hline $1444=5,988$ & Estepa & 158 & 77v (II) \\
\hline $4165=14,1036$ & Tarragona & 71 & $77 \mathrm{r}(\mathrm{I})$ \\
\hline $1459=5,913$ & Alameda & 159 & $77 \mathrm{r}(\mathrm{I})$ \\
\hline $1460=5,915$ & Alameda & 159 & $77 \mathrm{r}(\mathrm{I})$ \\
\hline 2335 & Peñaflor & 165 & $77 \mathrm{r}(\mathrm{I})$ \\
\hline
\end{tabular}

33 La inscripción estaba escrita justo al borde inferior de la hoja, que se ha perdido totalmente excepto la localización Gadib. 


\begin{tabular}{|l|l|l|l|}
\hline $3377=5,2$ & La Guardia & 169 & $77 \mathrm{r}(\mathrm{I})$ \\
\hline $3362=5,29$ & Jaén & 171 & $77 \mathrm{r}(\mathrm{I})$ \\
\hline
\end{tabular}

Inscripciones de Pons d'Icart (Herzog-August-Bibliothek, Guelf. 20.11 Aug. $4^{\circ}$ )

\begin{tabular}{|l|l|l|l|}
\hline CIL II $=\mathrm{II}^{2}$ & Lugar & Icart & Agustín \\
\hline $4427=14,1089$ & Tarragona & $53 \mathrm{r}-\mathrm{v}$ & $78 \mathrm{r}(\mathrm{II})$ \\
\hline $4164=14,1056$ & Tarragona & $54 \mathrm{r}$ & $78 \mathrm{r}(\mathrm{II})$ \\
\hline $4127=14,993$ & Tarragona & $54 \mathrm{v}$ & $78 \mathrm{r}(\mathrm{II})$ \\
\hline $4119=14,980$ & Tarragona & $55 \mathrm{r}$ & $78 \mathrm{r}(\mathrm{II})$ \\
\hline $4266=14,1016$ & Tarragona & $55 \mathrm{v}$ & $78 \mathrm{r}(\mathrm{II})$ \\
\hline $4221=14,1188$ & Tarragona & $56 \mathrm{r}$ & $78 \mathrm{r}(\mathrm{II})$ \\
\hline $4312=14,816$ & Tarragona & $56 \mathrm{v}$ & $78 \mathrm{r}(\mathrm{II})$ \\
\hline $4255=14,1174$ & Tarragona & $57 \mathrm{r}$ & $78 \mathrm{r}(\mathrm{II})$ \\
\hline $4121=14,984$ & Tarragona & $57 \mathrm{v}$ & $78 \mathrm{r}(\mathrm{II})$ \\
\hline $4243=14,1163$ & Tarragona & $58 \mathrm{v}$ & $78 \mathrm{r}(\mathrm{II})$ \\
\hline $4191=14,1112$ & Tarragona & $58 \mathrm{v}$ & $78 \mathrm{v}(\mathrm{I})$ \\
\hline $4195=14,1116$ & Tarragona & $59 \mathrm{v}$ & $78 \mathrm{v}(\mathrm{I})$ \\
\hline $4237=14,1159$ & Tarragona & $60 \mathrm{r}$ & $78 \mathrm{v}(\mathrm{I})$ \\
\hline $4192=14,1191$ & Tarragona & $60 \mathrm{v}$ & $78 \mathrm{v}(\mathrm{I})$ \\
\hline $4197=14,1118$ & Tarragona & $61 \mathrm{v}$ & $78 \mathrm{v}(\mathrm{I})$ \\
\hline $4242=14,1183$ & Tarragona & $62 \mathrm{r}$ & $78 \mathrm{v}(\mathrm{I})$ \\
\hline $4207=14,1129$ & Tarragona & $62 \mathrm{v}$ & $78 \mathrm{v}(\mathrm{I})$ \\
\hline $4269=14,1026$ & Tarragona & $63 \mathrm{r}$ & $78 \mathrm{v}(\mathrm{I})$ \\
\hline $4201=14,1192$ & Tarragona & $64 \mathrm{r}$ & $78 \mathrm{v}(\mathrm{I})$ \\
\hline $4071=14,819$ & Tarragona & $64 \mathrm{v}$ & $78 \mathrm{v}(\mathrm{I})$ \\
\hline $4196=14,1117$ & Tarragona & $65 \mathrm{r}$ & $78 \mathrm{v}(\mathrm{I})$ \\
\hline $4246=14,1184$ & Tarragona & $65 \mathrm{v}$ & $78 \mathrm{v}(\mathrm{I})$ \\
\hline $4189=14,111$ & Tarragona & $78 \mathrm{v}(\mathrm{II})$ \\
\hline $4110=14,971$ & Tarragona & $78 \mathrm{v}(\mathrm{II})$ \\
\hline $4407=14,1698$ & Tarragona & $78 \mathrm{v}(\mathrm{II})$ \\
\hline $4314=14,1281$ & Tarragona & $78 \mathrm{v}(\mathrm{II})$ \\
\hline $4278=14,1233$ & Tarragona & $68 \mathrm{r}$ & $78 \mathrm{v}(\mathrm{II})$ \\
\hline $4181=14,1094$ & Tarragona & $69 \mathrm{r}$ & $78 \mathrm{v}(\mathrm{II})$ \\
\hline $4165=14,1036$ & Tarragona & $69 \mathrm{v}$ & $78 \mathrm{v}(\mathrm{II})$ \\
\hline $4231=14,1155$ & Tarragona & $70 \mathrm{r}$ & $78 \mathrm{v}(\mathrm{II})$ \\
\hline $4270=14,1221$ & Tarragona & $70 \mathrm{v}$ & $78 \mathrm{r}(\mathrm{I})$ \\
\hline $4090=14,855$ & Tarragona & $78 \mathrm{r}(\mathrm{I})$ \\
\hline
\end{tabular}




\begin{tabular}{|c|c|c|c|}
\hline $4355=14,1311$ & Tarragona & $71 \mathrm{r}$ & $78 \mathrm{r}(\mathrm{I})$ \\
\hline $4275=14,1213$ & Tarragona & $71 \mathrm{v}$ & 78r (I) \\
\hline $4276=14,1224$ & Tarragona & $72 \mathrm{r}$ & $78 \mathrm{r}(\mathrm{I})$ \\
\hline $4393=14,1338$ & Tarragona & $72 v$ & 78r (I) \\
\hline $4356=14,1324$ & Tarragona & $72 v$ & 78r (I) \\
\hline $4268=14,1210$ & Tarragona & $73 r$ & $78 \mathrm{r}(\mathrm{I})$ \\
\hline $4261=14,1202$ & Tarragona & $73 v$ & $78 \mathrm{r}(\mathrm{I})$ \\
\hline $4174=14,1308$ & Tarragona & $74 r$ & $78 \mathrm{r}(\mathrm{I})$ \\
\hline $4083=14,839$ & Tarragona & $74 v$ & 78r (I) \\
\hline $4248=14,1194$ & Tarragona & $75 r$ & $78 \mathrm{r}(\mathrm{I})$ \\
\hline $4229=14,1153$ & Tarragona & $75 \mathrm{v}$ & 79r (II) \\
\hline $4166=14,994$ & Tarragona & $76 r$ & 79r (II) \\
\hline $4297=14,1253$ & Tarragona & $76 \mathrm{v}$ & 79r (II) \\
\hline $4227=14,1150$ & Tarragona & $77 \mathrm{r}$ & 79r (II) \\
\hline $4257=14,1168$ & Tarragona & $77 \mathrm{v}$ & 79r (II) \\
\hline $4099=14,907$ & Tarragona & $78 \mathrm{r}$ & 79r (II) \\
\hline $4097=14,911$ & Tarragona & $78 v$ & 79r (II) \\
\hline $4238=14,1160$ & Tarragona & $79 \mathrm{r}$ & 79r (II) \\
\hline $4352=14,1328$ & Tarragona & $79 v$ & 79r (II) \\
\hline $4114=14,975$ & Tarragona & $80 \mathrm{r}$ & 79r (II) \\
\hline $4278=14,1232$ & Tarragona & $80 \mathrm{v}$ & $79 \mathrm{v}(\mathrm{I})$ \\
\hline $4107=14,945$ & Tarragona & $81 \mathrm{v}$ & $79 \mathrm{v}(\mathrm{I})$ \\
\hline $4104=14,932$ & Tarragona & $82 \mathrm{r}$ & $79 \mathrm{v}(\mathrm{I})$ \\
\hline $4332=14,1235$ & Tarragona & $83 r$ & $79 \mathrm{v}(\mathrm{I})$ \\
\hline $4108=14,944$ & Tarragona & $83 r$ & 79v (I) \\
\hline $4293=14,1251$ & Tarragona & $83 v$ & $79 \mathrm{v}(\mathrm{I})$ \\
\hline $4103=14,930$ & Tarragona & $84 r$ & $79 \mathrm{v}(\mathrm{I})$ \\
\hline $4139=14,1006$ & Tarragona & $84 \mathrm{v}$ & $79 \mathrm{v}(\mathrm{I})$ \\
\hline $4103=14,930$ & Tarragona & $85 \mathrm{v}$ & $79 \mathrm{v}(\mathrm{I})$ \\
\hline $4108=14,944$ & Tarragona & $86 r$ & $79 \mathrm{v}(\mathrm{I})$ \\
\hline $4224=14,1019$ & Tarragona & $86 \mathrm{v}$ & 79v (II) \\
\hline $4102=14,929$ & Tarragona & $87 \mathrm{r}$ & 79v (II) \\
\hline $4105=14,939$ & Tarragona & $87 \mathrm{v}$ & 79v (II) \\
\hline $4106=14,942$ & Tarragona & $88 \mathrm{r}$ & $79 \mathrm{v}(\mathrm{II})$ \\
\hline $4126=14,990$ & Tarragona & $88 \mathrm{v}$ & $79 \mathrm{v}(\mathrm{II})$ \\
\hline $4089=14,853$ & Tarragona & $90 \mathrm{v}$ & $79 \mathrm{v}(\mathrm{II})$ \\
\hline $4403=14,1346$ & Tarragona & $91 \mathrm{r}$ & $79 \mathrm{v}(\mathrm{II})$ \\
\hline
\end{tabular}




\begin{tabular}{|c|c|c|c|}
\hline $4144=14,1045$ & Tarragona & $91 \mathrm{v}$ & 79v (II) \\
\hline $4130=14,999$ & Tarragona & $92 \mathrm{r}$ & 79v (II) \\
\hline $4264=14,1013$ & Tarragona & $92 \mathrm{r}$ & 79v (II) \\
\hline $4204=14,1126$ & Tarragona & $92 \mathrm{v}$ & $79 \mathrm{v}(\mathrm{II})$ \\
\hline $4136=14,1008$ & Tarragona & $93 r$ & 79r (I) \\
\hline $4390=14,1619$ & Tarragona & $93 v$ & 79r (I) \\
\hline $4226=14,1148$ & Tarragona & $94 \mathrm{r}$ & 79r (I) \\
\hline $4135=14,1002$ & Tarragona & $94 v$ & 79r (I) \\
\hline $4430=14,1344$ & Tarragona & $95 \mathrm{v}$ & 79r (I) \\
\hline $4198=14,1177$ & Tarragona & $95 \mathrm{v}$ & 79r (I) \\
\hline $4162=14,1035$ & Tarragona & $96 r$ & 79r (I) \\
\hline $4185=14,1106$ & Tarragona & $97 \mathrm{r}$ & 79r (I) \\
\hline $4080=14,827$ & Tarragona & $97 \mathrm{v}$ & 79r (I) \\
\hline $4395=14,1340$ & Tarragona & $98 \mathrm{r}$ & 79r (I) \\
\hline $4074=14,821$ & Tarragona & $98 \mathrm{r}$ & 79r (I) \\
\hline $4228=14,1151$ & Tarragona & $98 v$ & 79r (I) \\
\hline $4161=14,1083$ & Tarragona & $99 \mathrm{r}$ & 79r (I) \\
\hline $4313=14,1280$ & Tarragona & $99 \mathrm{v}$ & $80 \mathrm{r}$ (II) \\
\hline $4376=14,1590$ & Tarragona & $99 \mathrm{v}$ & 80r (II) \\
\hline $4120=14,983$ & Tarragona & $100 \mathrm{r}$ & 80r (II) \\
\hline $4378=14,1592$ & Tarragona & $100 \mathrm{r}$ & 80r (II) \\
\hline $4147=14,1031$ & Tarragona & $101 \mathrm{r}$ & 80r (II) \\
\hline $4406=14,1687$ & Tarragona & $101 \mathrm{v}$ & $80 \mathrm{r}$ (II) \\
\hline $4184=14,1096$ & Tarragona & $102 \mathrm{r}$ & 80r (II) \\
\hline $4362=14,1537$ & Tarragona & $103 r$ & $80 \mathrm{r}(\mathrm{II})$ \\
\hline $4337=14,1479$ & Tarragona & $110 \mathrm{v}$ & 80r (II) \\
\hline $4315=14,1285$ & Tarragona & $103 r$ & $80 \mathrm{v}(\mathrm{I})$ \\
\hline $4317=14,1289$ & Tarragona & $104 \mathrm{r}$ & $80 \mathrm{v}(\mathrm{I})$ \\
\hline $4372=14,1574$ & Tarragona & $104 \mathrm{v}$ & $80 \mathrm{v}(\mathrm{I})$ \\
\hline $4374=14,1578$ & Tarragona & $104 \mathrm{v}$ & $80 \mathrm{v}(\mathrm{I})$ \\
\hline $4128=14,995$ & Tarragona & $105 r$ & $80 \mathrm{v}(\mathrm{I})$ \\
\hline $4145=14,1047$ & Tarragona & $105 \mathrm{v}$ & $80 \mathrm{v}(\mathrm{I})$ \\
\hline $4143=14,1043$ & Tarragona & $106 \mathrm{r}$ & $80 \mathrm{v}(\mathrm{II})$ \\
\hline $4348=14,1501$ & Tarragona & $107 \mathrm{r}$ & $80 \mathrm{v}$ (II) \\
\hline $4262=14,1203$ & Tarragona & $107 \mathrm{v}$ & $80 \mathrm{v}(\mathrm{II})$ \\
\hline $4279=14,1217$ & Tarragona & $108 \mathrm{r}$ & $80 \mathrm{v}$ (II) \\
\hline $4141=14,1018$ & Tarragona & $108 \mathrm{v}$ & $80 \mathrm{v}(\mathrm{II})$ \\
\hline
\end{tabular}




\begin{tabular}{|c|c|c|c|}
\hline $4310=14,1286$ & Tarragona & $109 \mathrm{r}$ & $80 \mathrm{v}$ (II) \\
\hline $4388=14,1337$ & Tarragona & $109 \mathrm{r}$ & $80 \mathrm{v}$ (II) \\
\hline $4155=14,1039$ & Tarragona & $109 \mathrm{r}$ & $80 \mathrm{v}$ (II) \\
\hline $4405=14,1347$ & Tarragona & $109 \mathrm{v}$ & $80 \mathrm{v}$ (II) \\
\hline $4319=14,1277$ & Tarragona & $109 \mathrm{v}$ & $80 \mathrm{v}$ (II) \\
\hline $4233=14,1180$ & Tarragona & $110 \mathrm{r}$ & $80 \mathrm{v}$ (II) \\
\hline $4142=14,1064$ & Tarragona & $110 \mathrm{v}$ & $80 \mathrm{v}$ (II) \\
\hline $4118=14,979$ & Tarragona & $111 \mathrm{r}$ & $80 \mathrm{v}$ (II) \\
\hline $4154=14,1054$ & Tarragona & $112 \mathrm{r}$ & 80r (I) \\
\hline $4379=14,1593$ & Tarragona & $112 \mathrm{v}$ & 80r (I) \\
\hline $4418=14,1690$ & Tarragona & $113 r$ & $80 \mathrm{r}(\mathrm{I})$ \\
\hline $4361=14,1544$ & Tarragona & $113 \mathrm{v}$ & 80r (I) \\
\hline $4115=14,978$ & Tarragona & $114 \mathrm{r}$ & $80 \mathrm{r}(\mathrm{I})$ \\
\hline $4219=14,1141$ & Tarragona & $115 r$ & $80 \mathrm{r}(\mathrm{I})$ \\
\hline $4287=14,1238$ & Tarragona & $115 \mathrm{v}$ & 80r (I) \\
\hline $2227=14,1132$ & Tarragona & $115 \mathrm{v}$ & 80r (I) \\
\hline $4086 a=14,846$ & Tarragona & $116 \mathrm{r}$ & $80 \mathrm{r}(\mathrm{I})$ \\
\hline $4304=14,1262$ & Tarragona & $116 \mathrm{v}$ & $80 \mathrm{r}(\mathrm{I})$ \\
\hline $4148=14,1048$ & Tarragona & $116 \mathrm{v}$ & $80 \mathrm{r}(\mathrm{I})$ \\
\hline $4400=14,1657$ & Tarragona & $117 \mathrm{r}$ & 80r (I) \\
\hline $4410=14,1696$ & Tarragona & $117 \mathrm{~V}$ & 81 r (II) \\
\hline $4396=14,1647$ & Tarragona & $118 \mathrm{r}$ & $81 \mathrm{r}(\mathrm{II})$ \\
\hline $4325=14,1294$ & Tarragona & $118 \mathrm{r}$ & $81 \mathrm{r}(\mathrm{II})$ \\
\hline $4389=14,1613$ & Tarragona & $118 \mathrm{v}$ & $81 \mathrm{r}(\mathrm{II})$ \\
\hline $4385=14,1606$ & Tarragona & $119 \mathrm{r}$ & $81 \mathrm{r}(\mathrm{II})$ \\
\hline $4160=14,1055$ & Tarragona & $120 \mathrm{r}$ & $81 \mathrm{r}(\mathrm{II})$ \\
\hline $4366=14,1558$ & Tarragona & $120 \mathrm{v}$ & $81 \mathrm{r}(\mathrm{II})$ \\
\hline $4381=14,1587$ & Tarragona & $121 \mathrm{r}$ & $81 \mathrm{r}(\mathrm{II})$ \\
\hline $4156=14,1042$ & Tarragona & $121 \mathrm{r}$ & $81 \mathrm{r}(\mathrm{II})$ \\
\hline $4383=14,1599$ & Tarragona & $121 \mathrm{v}$ & 81 r (II) \\
\hline $4260=14,1195$ & Tarragona & $122 \mathrm{r}$ & $81 \mathrm{r}(\mathrm{II})$ \\
\hline $4432=14,865$ & Tarragona & $122 \mathrm{r}$ & 81 r (II) \\
\hline $4132=14,1024$ & Tarragona & $122 \mathrm{v}$ & $81 \mathrm{r}(\mathrm{II})$ \\
\hline $4336=14,1312$ & Tarragona & $122 \mathrm{v}$ & $81 \mathrm{r}(\mathrm{II})$ \\
\hline $4158=14,1034$ & Tarragona & $123 \mathrm{v}$ & $81 \mathrm{v}(\mathrm{I})$ \\
\hline $4414=14,1704$ & Tarragona & $124 \mathrm{r}$ & $81 \mathrm{v}(\mathrm{I})$ \\
\hline $4380=14,1586$ & Tarragona & $124 \mathrm{v}$ & $81 v(\mathrm{I})$ \\
\hline
\end{tabular}




\begin{tabular}{|c|c|c|c|}
\hline $4318=14,1273$ & Tarragona & $125 r$ & $81 \mathrm{v}(\mathrm{I})$ \\
\hline $4253=14,1172$ & Tarragona & $125 \mathrm{r}$ & $81 \mathrm{v}(\mathrm{I})$ \\
\hline $4121=14,984$ & Tarragona & $125 \mathrm{v}$ & $81 \mathrm{v}(\mathrm{I})$ \\
\hline $4289=14,1243$ & Tarragona & $126 \mathrm{v}$ & $81 v(\mathrm{I})$ \\
\hline $4335=14,1473$ & Tarragona & $127 \mathrm{v}$ & $81 v(\mathrm{I})$ \\
\hline $4072=14,822$ & Tarragona & $127 \mathrm{v}$ & $81 v(\mathrm{I})$ \\
\hline $4202=14,1124$ & Tarragona & $128 \mathrm{r}$ & $81 \mathrm{v}(\mathrm{I})$ \\
\hline $4398=14,1649$ & Tarragona & $128 \mathrm{v}$ & $81 \mathrm{v}(\mathrm{I})$ \\
\hline $4321=14,1299$ & Tarragona & $128 \mathrm{v}$ & $81 v(\mathrm{I})$ \\
\hline $4180=14,1090$ & Tarragona & $129 \mathrm{r}$ & $81 v(\mathrm{I})$ \\
\hline $4125=14,989$ & Tarragona & $129 \mathrm{v}$ & $81 \mathrm{v}(\mathrm{I})$ \\
\hline $4408=14,1699$ & Tarragona & $130 \mathrm{v}$ & $81 \mathrm{v}(\mathrm{I})$ \\
\hline $14, \mathrm{G} 2$ & Tarragona & $136 \mathrm{v}$ & $81 \mathrm{v}(\mathrm{I})$ \\
\hline $4271=14,1292$ & Tarragona & $130 \mathrm{v}$ & $81 \mathrm{v}$ (II) \\
\hline $4098=14,906$ & Tarragona & $131 \mathrm{r}$ & $81 \mathrm{v}$ (II) \\
\hline $4176=14,1076$ & Tarragona & $131 \mathrm{r}$ & $81 v$ (II) \\
\hline 14,1234 & Tarragona & $89 \mathrm{v}$ & 82 r (II) \\
\hline $14,2121=$ IHC 189 & Tarragona & $123 r$ & $82 \mathrm{r}$ (II) \\
\hline 14, 2132 = ICERV 219 & Tarragona & $123 \mathrm{v}$ & $82 \mathrm{r}(\mathrm{II})$ \\
\hline $4399=14,1653$ & Tarragona & $131 \mathrm{v}$ & $82 \mathrm{r}(\mathrm{II})$ \\
\hline $4360=14,1532$ & Tarragona & $131 \mathrm{v}$ & $82 \mathrm{r}(\mathrm{II})$ \\
\hline $4402=14,1661$ & Tarragona & $131 \mathrm{v}$ & $82 \mathrm{r}(\mathrm{II})$ \\
\hline $4392=14,1628$ & Tarragona & $132 \mathrm{r}$ & $82 \mathrm{r}(\mathrm{II})$ \\
\hline $4172=14,1086$ & Tarragona & $132 \mathrm{r}$ & $82 \mathrm{r}(\mathrm{II})$ \\
\hline $4300=14,2304$ & Tarragona [Mas Rabassa] & $132 \mathrm{v}$ & $82 \mathrm{r}(\mathrm{II})$ \\
\hline $4339=14,2330$ & Tarragona [Torredembarra] & $132 \mathrm{v}$ & $82 \mathrm{r}(\mathrm{II})$ \\
\hline $4117=14,2291$ & Tarragona [Villalonga del Camp] & $132 \mathrm{v}$ & $82 \mathrm{r}$ (II) \\
\hline $4446=14,2239$ & Tarragona [La Canonja] & $133 r$ & $82 \mathrm{r}(\mathrm{II})$ \\
\hline $4447=14,2240$ & Tarragona [La Canonja] & $133 r$ & $82 \mathrm{r}(\mathrm{II})$ \\
\hline $4170=14,2235$ & Tarragona [Vilaseca] & $133 \mathrm{v}$ & $82 \mathrm{r}$ (II) \\
\hline $4448=14,1325$ & Tarragona & $133 \mathrm{v}$ & $82 \mathrm{r}(\mathrm{II})$ \\
\hline $4199=14,1120$ & Tarragona & $134 \mathrm{r}$ & $82 \mathrm{r}(\mathrm{II})$ \\
\hline $4454=14,1249$ & Guissona [Tarragona] & $134 \mathrm{r}$ & $82 \mathrm{r}(\mathrm{II})$ \\
\hline $4455=14,1334$ & Guissona [Tarragona] & $134 \mathrm{v}$ & $82 \mathrm{r}(\mathrm{II})$ \\
\hline $4453=14,1246$ & Guissona [Tarragona] & $134 \mathrm{v}$ & $82 \mathrm{r}(\mathrm{II})$ \\
\hline 4452 & Guissona & $134 v$ & $82 \mathrm{v}(\mathrm{I})$ \\
\hline $4282=14,2332$ & Roda de Berà & $135 \mathrm{r}$ & $82 \mathrm{v}(\mathrm{I})$ \\
\hline
\end{tabular}




\begin{tabular}{|c|c|c|c|}
\hline $4091=14,858$ & Tarragona & $135 r$ & $82 \mathrm{v}(\mathrm{I})$ \\
\hline $4100=14,909$ & Tarragona & $135 r$ & $82 \mathrm{v}(\mathrm{I})$ \\
\hline $4087=14,850$ & Tarragona & $135 \mathrm{v}$ & $82 \mathrm{v}(\mathrm{I})$ \\
\hline $4329=14,1442$ & Tarragona & $135 \mathrm{v}$ & $82 \mathrm{v}(\mathrm{I})$ \\
\hline $4131=14,997$ & Tarragona & $135 \mathrm{v}$ & $82 \mathrm{v}(\mathrm{I})$ \\
\hline $4330=14,1455$ & Tarragona & $136 r$ & $82 \mathrm{v}(\mathrm{I})$ \\
\hline $4439=14,1313$ & Tarragona & $136 \mathrm{v}$ & $82 \mathrm{v}(\mathrm{I})$ \\
\hline $4397=14,1341$ & Tarragona & $136 \mathrm{v}$ & $82 \mathrm{v}(\mathrm{I})$ \\
\hline $4322=14,1033$ & Tarragona & $136 \mathrm{v}$ & $82 \mathrm{v}(\mathrm{I})$ \\
\hline $4349=14,1503$ & Tarragona & $137 \mathrm{r}$ & $82 \mathrm{v}(\mathrm{I})$ \\
\hline $4373=14,1576$ & Tarragona & $137 \mathrm{r}$ & $82 \mathrm{v}(\mathrm{I})$ \\
\hline $4288=14,1240$ & Tarragona & $137 \mathrm{v}$ & $82 \mathrm{v}(\mathrm{I})$ \\
\hline $4256=14,1189$ & Tarragona & $137 \mathrm{v}$ & $82 \mathrm{v}(\mathrm{I})$ \\
\hline $4193=14,1113$ & Tarragona & $138 \mathrm{r}$ & $82 \mathrm{v}(\mathrm{I})$ \\
\hline $4138=14,1010$ & Tarragona & $140 \mathrm{r}$ & $82 \mathrm{v}(\mathrm{I})$ \\
\hline $4140=14,1122$ & Tarragona & $140 \mathrm{r}$ & $82 \mathrm{v}(\mathrm{I})$ \\
\hline $4412=14,1697$ & Tarragona & $140 \mathrm{v}$ & $82 \mathrm{v}(\mathrm{I})$ \\
\hline $4175=14,1065$ & Tarragona & $141 \mathrm{r}$ & $82 \mathrm{v}(\mathrm{I})$ \\
\hline $4394=14,1637$ & Tarragona & $141 \mathrm{r}$ & $82 \mathrm{v}(\mathrm{I})$ \\
\hline $4208=14,1193$ & Tarragona & $141 \mathrm{r}$ & $82 \mathrm{v}$ (II) \\
\hline $4420=14,1617$ & Tarragona & $141 \mathrm{v}$ & $82 \mathrm{v}$ (II) \\
\hline $4391=14,1624$ & Tarragona & $141 \mathrm{v}$ & $82 \mathrm{v}(\mathrm{II})$ \\
\hline $4152=14,1051$ & Tarragona & $142 \mathrm{r}$ & $82 \mathrm{v}$ (II) \\
\hline $4311=14,1283$ & Tarragona & $142 \mathrm{v}$ & $82 \mathrm{~V}(\mathrm{II})$ \\
\hline $4129=14,974$ & Tarragona & $143 r$ & $82 \mathrm{v}$ (II) \\
\hline$f i c t a^{34}$ & Tarragona & $143 r$ & $82 \mathrm{v}(\mathrm{II})$ \\
\hline $393^{*}=14,31^{*}$ & Tarragona & $143 \mathrm{v}$ & $82 \mathrm{v}$ (II) \\
\hline $4345=14,1327$ & Tarragona & $143 r$ & $82 \mathrm{v}$ (II) \\
\hline $14,1445 \mathrm{a}$ & Tarragona & $144 \mathrm{r}$ & $82 \mathrm{~V}$ (II) \\
\hline $4158=14,1034$ & Tarragona & $144 \mathrm{r}$ & $82 \mathrm{~V}$ (II) \\
\hline $4253=14,1172$ & Tarragona & $144 v$ & $82 \mathrm{v}$ (II) \\
\hline
\end{tabular}

34 Inscripción falsa sin entrada en el CIL II. Se trataría de un anillo con las iniciales M. T. C. S., que Icart interpreta como Marcus Tullius Cicero senator. En su Libro de las grandezas, Icart (1572, 198r-202r) discute profusamente sobre este anillo y la posible presencia de Cicerón en Tarragona y da por extenso las razones por las cuales Agustín afirma su falsedad junto a la del epígrafe CIL II 393*, que aparece a continuación en el manuscrito que analizamos. En la original versión catalana manuscrita de la obra de Icart Llibre de les grandeses de Tarragona, publicada por Duran 1984, el autor se refiere a la inscripción de forma mucho más sucinta. Cf. Duran 1984: 204-205. 


\begin{tabular}{|l|l|l|l|}
\hline $4355=14,1311$ & Tarragona & $145 \mathrm{r}$ & $82 \mathrm{v}(\mathrm{II})$ \\
\hline $383^{*}=14,43^{*}$ & Tarragona & $145 \mathrm{r}$ & $82 \mathrm{v}(\mathrm{II})$ \\
\hline $4092=14,857$ & Tarragona & $145 \mathrm{v}$ & $82 \mathrm{v}(\mathrm{II})$ \\
\hline $4167=14,1057$ & Tarragona & $145 \mathrm{v}$ & $82 \mathrm{v}(\mathrm{II})$ \\
\hline $4220=14,1142$ & Tarragona & $145 \mathrm{v}$ & $82 \mathrm{v}(\mathrm{II})$ \\
\hline $4414=14,1704$ & Tarragona & $146 \mathrm{r}$ & $82 \mathrm{v}(\mathrm{II})$ \\
\hline $4111=14,972$ & Tarragona & $146 \mathrm{r}$ & $82 \mathrm{v}(\mathrm{II})$ \\
\hline $4194=14,1115$ & Tarragona & $146 \mathrm{v}$ & $82 \mathrm{r}(\mathrm{I})$ \\
\hline $4345=14,1327$ & Tarragona & $146 \mathrm{v}$ & $82 \mathrm{r}(\mathrm{I})$ \\
\hline $4157=14,1067$ & Tarragona & $147 \mathrm{r}$ & $82 \mathrm{r}(\mathrm{I})$ \\
\hline $4307=14,1267$ & Tarragona & $149 \mathrm{r}$ & $82 \mathrm{r}(\mathrm{I})$ \\
\hline $4414=14,1704$ & Tarragona & $149 \mathrm{v}$ & $82 \mathrm{r}(\mathrm{I})$ \\
\hline $4383=14,1599$ & Tarragona & $149 \mathrm{v}$ & $82 \mathrm{r}(\mathrm{I})$ \\
\hline 14,1256 & Tarragona & $150 \mathrm{r}$ & $82 \mathrm{r}(\mathrm{I})$ \\
\hline $3892=14,432$ & Sagunto & $150 \mathrm{r}$ & $82 \mathrm{r}(\mathrm{I})$ \\
\hline $383 / 4=14,315 / 6$ & Sagunto & $150 \mathrm{r}$ & $82 \mathrm{r}(\mathrm{I})$ \\
\hline $3860=14,362$ & Sagunto & $150 \mathrm{v}$ & $82 \mathrm{r}(\mathrm{I})$ \\
\hline $3840=14,333$ & Sagunto & $150 \mathrm{v}$ & $82 \mathrm{r}(\mathrm{I})$ \\
\hline $4321=14,1299$ & Tarragona & $150 \mathrm{v}$ & $82 \mathrm{r}(\mathrm{I})$ \\
\hline $4413=14,1705$ & Tarragona & $151 \mathrm{r}$ & $82 \mathrm{r}(\mathrm{I})$ \\
\hline ficta 35 & Salou & $151 \mathrm{r}$ & $82 \mathrm{r}(\mathrm{I})$ \\
\hline $3837=14,329$ & Salou [Sagunto] & $151 \mathrm{v}$ & $82 \mathrm{r}(\mathrm{I})$ \\
\hline
\end{tabular}

35 Inscripción falsa sin entrada en el CIL II. Icart la localiza «prop la torre de Salou en una gran pedra prop lo cami», y el texto empieza: Dardanio Erictonio Troo / Ganimede... 
Inscripciones tarraconenses «ex Jo. Hermang. et Bux.»"

\begin{tabular}{|c|c|c|c|c|}
\hline $\mathrm{CIL} \mathrm{II} \mathrm{=} \mathrm{II}^{2}$ & Lugar & Joan Armengol $^{36}$ & Bux. & Agustín \\
\hline $4071=14,819$ & Tarragona & Vic. $179 \mathrm{v}=6039$ 206r & - & $81 \mathrm{v}$ (II) \\
\hline 14,1345 & Tarragona & Vic. $180 \mathrm{r}=6039206 \mathrm{v}$ & - & $81 \mathrm{v}$ (II) \\
\hline $4111=14,972$ & Tarragona & Vic. $183 r=6039206 \mathrm{v}$ & - & $81 \mathrm{v}$ (II) \\
\hline $4092=14,857$ & Tarragona & Vic. $179 \mathrm{v}=6039207 \mathrm{r}$ & - & $81 v$ (II) \\
\hline $4345=14,1327$ & Tarragona & Vic. $187 \mathrm{r}=6039210 \mathrm{v}$ & - & $81 \mathrm{v}$ (II) \\
\hline $4151=14,1032$ & $\begin{array}{l}\text { Barcelona }[\mathrm{Ta}- \\
\text { rragona }]\end{array}$ & Vic. $189 \mathrm{v}=6039208 \mathrm{r}$ & - & $81 v$ (II) \\
\hline $4339=14,2330$ & [Torredembarra] & Vic. $190 \mathrm{v}=6039208 \mathrm{r}$ & - & $81 \mathrm{v}$ (II) \\
\hline $4413=14,1705$ & Tarragona & Vic. $190 \mathrm{v}=6039208 \mathrm{v}$ & - & $81 \mathrm{v}$ (II) \\
\hline $4445=14,2238$ & [La Canonja] & Vic. $191 \mathrm{r}=6039208 \mathrm{v}$ & - & $81 \mathrm{v}$ (II) \\
\hline $4157=14,1067$ & Tarragona & Vic. $196 \mathrm{v}=6039209 \mathrm{r}$ & - & $81 \mathrm{v}$ (II) \\
\hline $4370=14,1565$ & Tarragona & Vic. $191 \mathrm{v}=6039208 \mathrm{v}$ & - & $81 \mathrm{v}$ (II) \\
\hline $4397=14,1341$ & Tarragona & Vic. 186r $=6039209 r$ & - & $81 \mathrm{r}(\mathrm{I})$ \\
\hline $4395=14,1340$ & Tarragona & $6039209 \mathrm{r}$ & - & $81 \mathrm{r}(\mathrm{I})$ \\
\hline $4276=14,1224$ & Tarragona & $6039209 \mathrm{r}$ & - & $81 \mathrm{r}(\mathrm{I})$ \\
\hline $386^{*}=14,21^{*}$ & Tarragona & - & L. Parm. 2r & $81 \mathrm{r}(\mathrm{I})$ \\
\hline $388^{*}=14,24 *$ & Tarragona & - & L. Parm. $2 \mathrm{r}$ & $81 \mathrm{r}(\mathrm{I})$ \\
\hline $387 a^{*}=14,23 *$ & Tarragona & - & L. Parm. 2v & $81 \mathrm{r}(\mathrm{I})$ \\
\hline $385^{*}=14,19^{*}$ & Tarragona & - & L. Parm. 2v & $81 \mathrm{r}(\mathrm{I})$ \\
\hline $4304=14,1262$ & Tarragona & - & $\begin{array}{l}\text { L. Parm. 3r; } \\
\text { 3v }\end{array}$ & $81 \mathrm{r}(\mathrm{I})$ \\
\hline $404^{*}=14,1445 \mathrm{a}$ & Tarragona & - & L. Parm. 3v & $81 \mathrm{r}(\mathrm{I})$ \\
\hline $4381=14,1587$ & Tarragona & - & L. Parm. $15 \mathrm{r}$ & $81 \mathrm{r}(\mathrm{I})$ \\
\hline $4383=14,1599$ & Tarragona & - & L. Parm. $15 \mathrm{r}$ & $81 \mathrm{r}(\mathrm{I})$ \\
\hline $4168=14,1059$ & Tarragona & - & L. Parm. $15 \mathrm{v}$ & $81 \mathrm{r}(\mathrm{I})$ \\
\hline $4167=14,1057$ & Tarragona & - & L. Parm. $15 \mathrm{v}$ & $81 \mathrm{r}(\mathrm{I})$ \\
\hline
\end{tabular}

Inscripciones de fuente no identificada (¿Obra perdida de Fernández Franco?)

\begin{tabular}{|l|l|l|}
\hline CIL II $=$ II $^{2}$ & Lugar & Agustín \\
\hline $1578=5,398$ & Castro del Río & 72 r (II) \\
\hline $1575=5,395$ & Castro del Río & 72 r (II) \\
\hline $1581=5,400$ & Castro del Río & 72 r (II) \\
\hline
\end{tabular}

36 No se ha conservado la síloge original de Armengol; damos como referencia la síloge de Francesc Vicent (Wolfenbüttel, Herzog-August-Bibliothek, cod. Guelf. 20.11.Aug. 4º, ff. 179r-222v), de la que deriva en gran medida (véase supra), y la sección de la síloge de Jean Matal procedente ab Ioanne Hermangolio Tarraconensi (Biblioteca Apostolica Vaticana, ms. Vat. lat. 6039, ff. 206r-211v). 


\begin{tabular}{|l|l|l|}
\hline $1579=5,397$ & Castro del Río & $72 \mathrm{r}(\mathrm{II})$ \\
\hline $1576=5,458$ & Espejo & $72 \mathrm{r}(\mathrm{II})$ \\
\hline $1570=5,401$ & Castro del Río & $72 \mathrm{r}(\mathrm{II})$ \\
\hline $1569=5,394$ & Castro del Río & $72 \mathrm{r}(\mathrm{II})$ \\
\hline $2141=7,111$ & Porcuna & $72 \mathrm{r}(\mathrm{II})$ \\
\hline $2130=7,126$ & Porcuna & $72 \mathrm{r}(\mathrm{II})$ \\
\hline 1733 & Cádiz [Jerez] & $72 \mathrm{v}(\mathrm{II})$ \\
\hline $1405=5,1027$ & Osuna & $72 \mathrm{v}(\mathrm{II})$ \\
\hline $1404=5,1025$ & Osuna & $72 \mathrm{v}(\mathrm{II})$ \\
\hline 4695 & Archidona & $72 \mathrm{v}(\mathrm{II})$ \\
\hline 1969 & Málaga & $72 \mathrm{v}(\mathrm{II})$ \\
\hline
\end{tabular}

Inscripciones de fuente no identificada (de Andalucía y Vila Viçosa)

\begin{tabular}{|c|c|c|}
\hline $\mathrm{CIL} \mathrm{II} \mathrm{=} \mathrm{II}^{2}$ & Lugar & Agustín \\
\hline $2070=5,620$ & Granada & $75 \mathrm{v}(\mathrm{I})$ \\
\hline $2206=7,265$ & Córdoba & $75 \mathrm{v}(\mathrm{I})$ \\
\hline $2156=7,139$ & Montoro & $75 \mathrm{v}(\mathrm{I})$ \\
\hline $2111=7,74$ & Arjona & $75 \mathrm{v}(\mathrm{I})$ \\
\hline 3336 & Baeza & $75 \mathrm{v}(\mathrm{I})$ \\
\hline 149 & Vila Viçosa & $75 \mathrm{v}(\mathrm{I})$ \\
\hline 148 & Vila Viçosa & $75 \mathrm{v}(\mathrm{I})$ \\
\hline 144 & Vila Viçosa & $75 \mathrm{v}(\mathrm{I})$ \\
\hline 129 & Vila Viçosa & 75v (II) \\
\hline 128 & Vila Viçosa & $75 \mathrm{v}$ (II) \\
\hline 139 & Vila Viçosa & $75 \mathrm{v}$ (II) \\
\hline 143 & Vila Viçosa & 75v (II) \\
\hline 142 & Vila Viçosa & 75v (II) \\
\hline 137 & Vila Viçosa & 75v (II) \\
\hline 127 & Vila Viçosa & 75v (II) \\
\hline 131 & Vila Viçosa & 75v (II) \\
\hline HEp 1993, 477 & Vila Viçosa & 75v (II) \\
\hline 135 & Vila Viçosa & 75v (II) \\
\hline 130 & Vila Viçosa & 75v (II) \\
\hline 132 & Vila Viçosa & 75v (II) \\
\hline 136 & Vila Viçosa & $75 \mathrm{v}(\mathrm{II})$ \\
\hline 138 & Vila Viçosa & $75 \mathrm{v}$ (II) \\
\hline
\end{tabular}


Inscripciones saguntinas de fuente no identificada

\begin{tabular}{|c|c|c|c|}
\hline $\mathrm{CIL} \mathrm{II}=\mathrm{II}^{2}$ & Lugar & Agustín & Martín de Bailo \\
\hline $3871=14,347$ & Sagunto & 76r (II) & \\
\hline $3850=14,346$ & Sagunto & $76 r(I)$ & \\
\hline $\begin{array}{c}3848-9=14 \\
344-5\end{array}$ & Sagunto & 76r (I) & \\
\hline $3847=14,343$ & Sagunto & 76r (I) & \\
\hline $3846=14,342$ & Sagunto & 76r (I) & \\
\hline $3840=14,333$ & Sagunto & 76r (I) & \\
\hline $3865=14,365$ & Sagunto & $76 \mathrm{r}(\mathrm{I})$ & \\
\hline $3866=14,367$ & Sagunto & $76 r(I)$ & \\
\hline $3850=14,346$ & Sagunto & & $76 \mathrm{r}(\mathrm{I})$ \\
\hline $3845=14,341$ & Sagunto & & 76r (I) \\
\hline $3851=14,335$ & Sagunto & & 76v (II) \\
\hline $3838=14,330$ & Sagunto & & 76v (II) \\
\hline $3912=14,475$ & Sagunto & & 76v (II) \\
\hline $3858=14,357$ & Sagunto & & 76v (II) \\
\hline $3860=14,362$ & Sagunto & & 76v (II) \\
\hline $3837=14,329$ & Sagunto & & 76v (II) \\
\hline $3842=14,338$ & Sagunto & & $75 \mathrm{v}$ (II) \\
\hline $3843=14,339$ & Sagunto & & $75 \mathrm{v}$ (II) \\
\hline $3844=14,340$ & Sagunto & & $75 \mathrm{v}$ (II) \\
\hline $3841=14,337$ & Sagunto & & $75 \mathrm{v}$ (II) \\
\hline $3832=14,317$ & Sagunto & & $75 \mathrm{v}$ (II) \\
\hline $3916=14,478$ & Sagunto & & $75 \mathrm{v}(\mathrm{II})$ \\
\hline $3892=14,432$ & Sagunto & & $75 \mathrm{v}$ (II) \\
\hline $3940=14,533$ & Sagunto & & $75 \mathrm{v}$ (II) \\
\hline $3835=14,318$ & Sagunto & & $75 \mathrm{v}$ (II) \\
\hline $3856=14,354$ & Sagunto & & $75 \mathrm{v}$ (II) \\
\hline $3921=14,485$ & Sagunto & & $75 \mathrm{v}$ (II) \\
\hline $3825=14,298$ & Sagunto & & $75 \mathrm{v}$ (II) \\
\hline $3859=14,359$ & Sagunto & & $75 \mathrm{v}$ (II) \\
\hline $3914=14,479$ & Sagunto & & $75 \mathrm{v}$ (II) \\
\hline $3860=14,362$ & Sagunto & & $75 \mathrm{v}$ (II) \\
\hline
\end{tabular}




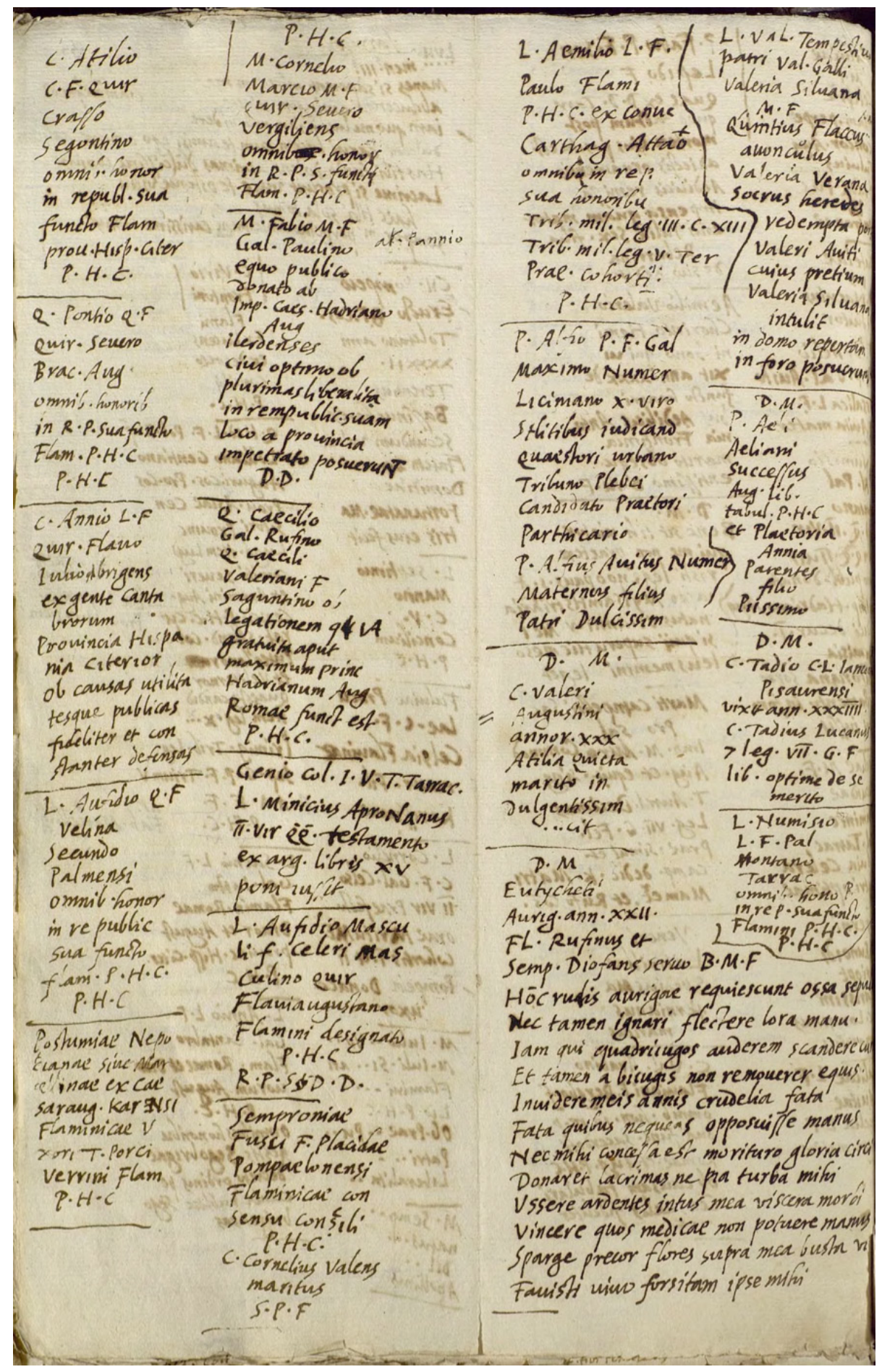

Fig. 1. BNE, ms. 5781 f. $78 \mathrm{v}$ 


\section{Bibliografía}

Agustín, A. (1765-1774), Opera Omnia, VIII vols., Lucca, typis Josephi Rocchii.

Cahner, M. (1978), Epistolari del Renaixement, II, Valencia, Clàssics Albatros.

Carbonell, J. (1991), Epigrafia i numismàtica a l'epistolari d'Antonio Agustín (1551 - 1563), tesis doctoral, Bellaterra, Universitat Autònoma de Barcelona. $<$ http://www.tdx.cat/handle/10803/5551> [02/11/2017].

Carbonell, J. (1993), «L'identification des papiers d'Antonio Agustín à travers sa correspondance», en Crawford 1993, 113-132.

Carbonell, J. (2002), «Antonio Agustín, filólogo epigrafista: a propósito de la Lex agraria (CIL I ${ }^{2}$ 585)», en Maestre, J. M. - Pascual, J. - Charlo, L. (eds.), Humanismo y Pervivencia del mundo clásico III, I, Alcañiz-Madrid, Ediciones del Laberinto, 397-412.

Carbonell, J. (2005), «Filología, numismática y prosopografía. La síntesis de Antonio Agustín y Fulvio Orsini», en Alfaro, C.; Marcos, C.; Otero, P. (eds.), Actas del XIII Congreso Internacional de Numismática, Madrid, Ministerio de Cultura, 59-67.

Carbonell, J. (2009), «Ambientes humanísticos en Roma (1545-1555). El cenáculo de Ottavio Pantagato, Antonio Agustín y Jean Matal», en De la Mota, C. - Puigvert, G. (eds.), La investigación en Humanidades, Madrid, Biblioteca Nueva, 47-70.

Carbonell, J. - Gimeno, H. (2016), «Juan Fernández Franco, Antonio Agustín y la Epigrafía Bética. Nuevos datos para una monografía», en Carbonell, J. - Gimeno, H. (eds.), A Baete ad fluvium Anam: Cultura epigráfica en la Bética Occidental y territorios fronterizos, Alcalá de Henares, UAH, 267-288.

Carbonell, J. - Gimeno, H. - Vargas, G. (1992), «Las inscripciones de los Adversaria de Antonio Agustín del ms. 5781 (= Q 87) de la Biblioteca Nacional de Madrid», Epigraphica 54, 167-190.

Carbonell, J. - Soler, A. (2011), «La polémica de las asambleas romanas en el círculo de Antonio Agustín y Ottavio Pantagato», en De la Villa, J. - González Castro, J. F. - Hinojo, G. (eds.), Perfiles de Grecia y Roma. Actas del XII Congreso Español de Estudos Clásicos, III, Madrid, Sociedad Española de Estudios Clásicos, 195-207.

Cooper, R. (1993), «Epigraphical Research in Rome in the Mid-Sixteenth Century: The Papers of Antonio Agustín and Jean Matal», en Crawford 1993, 95-111.

Crawford, M. H. (ed.) (1993), Antonio Agustín Between Renaissance and Counter-Reform, London, The Warburg Institute-University of London.

Duran, E. (1984), Lluís Ponç d'Icard i el Llibre de les grandeses de Tarragona, Barcelona, Curial.

Duran, E. (2005), «Historiografia catalana del segle XVI. Els antiquaris», en Martínez Romero, T. (ed.), Les lletres hispàniques als segles XVI, XVII i XVIII, Castelló de la Plana, Servei de Publicacions de la Universitat Jaume I, 153-173.

Flores Sellés, C. (1980), Epistolario de Antonio Agustín, Salamanca, Ediciones de la Universidad.

Flores Sellés, C. (1982), Alveolus (Manuscrito Escurialense S-II-18), Madrid, Fundación Universitaria Española.

González Germain, G. (2011), Estudi i edició de les inscripcions llatines falses d'Hispània (ca. 1440-1550), tesis doctoral, Bellaterra, Universitat Autònoma de Barcelona. $<$ http:// hdl.handle.net/10803/83977> [02/11/2017].

González Germain, G. (2013), El despertar epigráfico en el Renacimiento hispánico, Corpora et manuscripta epigraphica saeculis XV et XVI, Faenza, Fratelli Lega.

González Germain, G. (2016), «La síloge de inscripciones del Liber Parmensis: un testi- 
monio inédito del estudio de la epigrafía en Barcelona hacia 1510», Sylloge epigraphica Barcinonensis 14, 21-45.

Gozalbes Cravioto, E. (2007), «Antigüedades romanas en los manuscritos del erudito Juan Fernández Franco (siglo XVI)», Antiquitas 18-19, 227-235.

Gual Vilà, V. (1992-1993), «Diverses notícies sobre els Armengol», Paratge 3-4, 77-87.

Hernando Sobrino, M. del R. (2009), Manuscritos de contenido epigráfico de la Biblioteca Nacional de Madrid (siglos XVI-XX). La transmisión de las inscripciones de la Hispania romana y visigoda, Madrid, Atenea, 2009 [2010].

Louzado, B. (1998), «Un florilegio de epitafios renacentistas en el ms. 5973 de la Biblioteca Nacional», Cuadernos de Filología Clásica. Estudios latinos 14, 207-221.

Massó, J. (1985), «Notes per a una bibliografia de Lluís Pons d'Icart (1518/20-1578)», Treballs Canongins, Reus, Centre d'Estudis Canongins Ponç de Castellví, 65-102.

Massó, J. (1989), «Notes sobre escultures i inscripcions romanes a la Tarragona dels segles XVI, XVII i XVIII», Faventia 11/1, 83-108.

Massó, J. (2003), «Lluís Pons d'Icart, vida i obra», en El Renaixement de Tàrraco 1563. Lluís Pons d'Icart i Anton Van den Wyngaerde, Tarragona, Museu Nacional Arqueològic de Catalunya, 27-41.

Mayer, M. (1984), «Epigrafía hispánica y transmisión literaria con especial atención a la manuscrita», en Étienne, R. (cur.), Épigraphie hispanique. Problèmes de méthode et d'édition, Paris, Publications du Centre Pierre Paris, 35-57.

Rovira i Gómez, S.-J. (2006), «Notes d'arxiu sobre els Armengol, barons de Rocafort de Queralt», Aplec de Treballs (Montblanc) 24, 109-116.

Vagenheim, G. (2006), «Manus epigraphicae. Pirro Ligorio et d'autres érudits dans les recueils d'inscriptions latines de Jean Matal», en Deramaix, M. - Vagenheim, G. (eds.), L'Italie et la France dans l'Europe latine du XIVe au XVIIe siècle: influence, émulation, traduction, Mont-Saint-Aignan, Publications des Universités de Rouen et du Havre, 233270. 